\title{
Study of electroweak vacuum stability from extended Higgs portal of dark matter and neutrinos
}

\author{
Purusottam Ghosh, ${ }^{*}$ Abhijit Kumar Saha, ${ }^{\dagger}$ and Arunansu Sil ${ }^{\ddagger}$ \\ Department of Physics, Indian Institute of Technology Guwahati, 781039 Assam, India
}

(Received 28 June 2017; published 26 April 2018)

\begin{abstract}
We investigate the electroweak vacuum stability in an extended version of the Standard Model that incorporates two additional singlet scalar fields and three right-handed neutrinos. One of these extra scalars plays the role of dark matter, while the other scalar not only helps make the electroweak vacuum stable but also opens up the low-mass window of the scalar singlet dark matter $(<500 \mathrm{GeV})$. We consider the effect of large neutrino Yukawa coupling on the running of Higgs quartic coupling. We have analyzed the constraints on the model and identified the range of parameter space that is consistent with the neutrino mass, appropriate relic density, and direct search limits from the latest XENON 1T preliminary result as well as realized the stability of the electroweak vacuum up to the Planck scale.
\end{abstract}

DOI: 10.1103/PhysRevD.97.075034

\section{INTRODUCTION}

The discovery of the Higgs boson [1-3] has been considered the greatest triumph in present-day particle physics. Although the experimental search is still on in order to investigate the Higgs boson's properties, several theoretical and phenomenological reasons are there to push us toward hunting for an enlarged Higgs sector compared to the one present in Standard model (SM). For example, the Higgs quartic coupling $\lambda_{H}$ in the SM becomes negative at a high-energy scale $\left(\Lambda_{I}^{\mathrm{SM}} \sim 10^{10} \mathrm{GeV}\right)$, leading to the possible instability of the Higgs vacuum. The present measured values of the Higgs mass, $125.09 \mathrm{GeV}$ [4], and top mass, $173.2 \mathrm{GeV}$ [4], suggest that the electroweak (EW) vacuum can be metastable [5-11]. However, the conclusion exclusively depends on the precise measurement of the top mass $[12,13]$. Also, the metastability of the Universe is not a very robust situation in the context of cosmological inflation [14]. One of the possible solutions to this is to introduce new physics between the EW scale and $\Lambda_{I}^{\mathrm{SM}}$. In view of the SM's incompetence in resolving some of the other issues like dark matter, neutrino mass, matterantimatter symmetry, inflation, etc., the introduction of new physics is, of course, a welcome feature.

\footnotetext{
*pghoshiitg@gmail.com abhijit.saha@iitg.ernet.in *asil@iitg.ernet.in
}

Published by the American Physical Society under the terms of the Creative Commons Attribution 4.0 International license. Further distribution of this work must maintain attribution to the author(s) and the published article's title, journal citation, and DOI. Funded by SCOAP .
In particular, the SM fails to accommodate a significant share in terms of its content called dark matter (DM). The most economical and popular scenario is the singlet scalar extension of the SM [15-23] having Higgs portal interaction. The stability of the dark matter is ensured by imposing a $Z_{2}$ symmetry on it. The relic abundance and corresponding direct detection cross section are solely determined by the DM (the scalar singlet) mass and its coupling with Higgs (portal coupling). However, present experiments, LUX [24] and XENON1T [25], strongly disfavor the model below $m_{\mathrm{DM}}<500 \mathrm{GeV}$ except the resonance region. The bound on Higgs invisible decay width further constrains the model for $m_{\mathrm{DM}}<62.5 \mathrm{GeV}$ [26]. Hence, a large range of DM mass seems to be excluded within this simplest framework, which otherwise would be an interesting region in which to search for several ongoing and future direct $[24,25,27]$ and indirect experiments [28]. It is interesting to note that the presence of an extra scalar in the form of DM can shift the instability scale $\left(\Lambda_{I}\right)$ toward a larger value compared to the SM one $\left(\Lambda_{I}>\Lambda_{I}^{\mathrm{SM}}\right)[29-35]$.

On the other hand, to accommodate nonzero neutrino mass via the type-I seesaw mechanism, one can extend the SM with three right-handed $(\mathrm{RH})$ neutrinos. The $\mathrm{RH}$ neutrinos, being the SM singlet, will have standard Yukawa-like coupling involving Higgs and lepton doublets. The presence of the neutrino Yukawa coupling affects the running of the Higgs quartic coupling similarly to the top Yukawa coupling. In fact, with neutrino Yukawa coupling, $Y_{\nu}$, of $\mathcal{O}(1), \Lambda_{I}$ could be lower than $\Lambda_{I}^{\mathrm{SM}}$ [36-46], which might lead to an unstable Universe. The situation is not altered much even if one includes scalar singlet DM $\left(m_{\mathrm{DM}} \leq 500 \mathrm{GeV}\right)$ in this framework $[34,36,47-50]$. 
So, the combined framework of RH neutrinos and scalar singlet DM excludes a significant range of DM mass $(<500 \mathrm{GeV})$ while keeping the EW vacuum on the verge of being unstable.

With an endeavor to make the EW vacuum absolutely stable up to the Planck scale $M_{P}$, in a scenario that can accommodate both the DM and massive neutrinos with large $Y_{\nu}$ (in type-I seesaw) and simultaneously reopen the window for lighter scalar singlet DM mass $(<500 \mathrm{GeV})$, we incorporate two SM real singlet scalars and three SM singlet RH neutrinos in this work.

Similar models to address DM phenomenology involving additional scalars (without involving RH neutrinos) have been studied [35,51-55], however with different motivations. Our setup also differs from them in terms of the inclusion of light neutrino mass through the type-I seesaw. The proposed model has several important ingredients that are mentioned below along with their importance:

(i) One of the additional SM singlet scalars is our DM candidate of which the stability is achieved with an unbroken $Z_{2}$ symmetry.

(ii) The other scalar would acquire a nonzero vacuum expectation value (vev). This field has twofold contributions in our analysis: i) it affects the running of the SM Higgs quartic coupling, and ii) the dark matter phenomenology becomes more involved due to its mixing with the SM Higgs and the DM.

(iii) The setup also contains three RH neutrinos in order to generate nonzero light neutrino mass through the type-I seesaw mechanism. Therefore, along with the contributions from the additional scalar fields, neutrino Yukawa coupling, $Y_{\nu}$, is also involved in studying the running of the Higgs quartic coupling.

We observe that the presence of the scalar $^{1}$ with a nonzero vev affects the DM phenomenology in such a way that $m_{\mathrm{DM}}$ less than $500 \mathrm{GeV}$ becomes a perfectly allowed mass range considering the recent XENON-1T result [25], which otherwise was excluded from the DM direct search results [62]. We also include the XENON-nT [25] prediction to further constrain our model. On the other hand, we find that the SM Higgs quartic coupling may remain positive until $M_{P}$ (or up to some other scale higher than $\Lambda_{I}^{\mathrm{SM}}$ ) even in the presence of large $Y_{\nu}$, thanks to the involvement of the scalar with a nonzero vev. We therefore identify the relevant parameter space (in terms of stability, metastability, and instability regions) of the model that can allow large $Y_{\nu}$ (with different mass scales of RH neutrinos) and scalar DM below $500 \mathrm{GeV}$. Bounds from other related aspects, e.g., lepton flavor-violating decays, neutrinoless double beta decay, etc., are also considered. The setup

\footnotetext{
${ }^{1}$ This scalar can perhaps be identified with moduli/inflaton fields [56-60] or a messenger field [61] connecting the SM and any hidden sector.
}

therefore demands rich phenomenology that we present in the following sections.

The paper is organized as follows. In Sec. II, we discuss the setup of our model, and in Sec. III, we include the constraints on our model parameters. Then, in the subsequent Secs. IV and V, we discuss the DM phenomenology and vacuum stability, respectively, in the context of our model. In Sec. VI, we discuss the connection of the model with other observables. Finally, we conclude in Sec. VII.

\section{MODEL}

As mentioned in the Introduction, we aim to study how the EW vacuum can be made stable in a model that would successfully accommodate a scalar DM and neutrino mass. For this purpose, we extend the SM by introducing two SM singlet scalar fields, $\phi$ and $\chi$, and three right-handed neutrinos, $N_{i=1,2,3}$. We have also imposed a discrete symmetry, $Z_{2} \times Z_{2}^{\prime}$. The field $\phi$ is odd (even) under $Z_{2}$ $\left(Z_{2}^{\prime}\right)$, and $\chi$ is even (odd) under $Z_{2}\left(Z_{2}^{\prime}\right)$, while all other fields are even under both $Z_{2}$ and $Z_{2}^{\prime}$. There exists a nonzero vev associated with the $\chi$ field. The unbroken $Z_{2}$ ensures the stability of our dark matter candidate $\phi$. On the other hand, the inclusion of $Z_{2}^{\prime}$ simplifies the scalar potential in the setup. ${ }^{2}$ The RH neutrinos are included in order to incorporate the light neutrino mass through the type-I seesaw mechanism.

The scalar potential involving $\phi, \chi$, and the SM Higgs doublet $(H)$ is given by

$$
V=V_{\mathrm{I}}+V_{\mathrm{II}}+V_{\mathrm{III}}+V_{\mathrm{H}},
$$

where

$$
\begin{aligned}
V_{\mathrm{I}} & =\frac{1}{2} \mu_{\phi}^{2} \phi^{2}+\frac{1}{4 !} \lambda_{\phi} \phi^{4}+\frac{1}{2} \lambda_{\phi H} \phi^{2} H^{\dagger} H ; \\
V_{\mathrm{II}} & =-\frac{1}{2} \mu_{\chi}^{2} \chi^{2}+\frac{\lambda_{\chi}}{4 !} \chi^{4}+\frac{\lambda_{\chi H}}{2} \chi^{2}|H|^{2} ; \\
V_{\mathrm{III}} & =\frac{1}{4} \lambda_{\chi \phi} \phi^{2} \chi^{2}, \quad \text { and } \quad V_{\mathrm{H}}=-\mu_{H}^{2} H^{\dagger} H+\lambda_{H}\left(H^{\dagger} H\right)^{2} .
\end{aligned}
$$

The relevant part of the Lagrangian responsible for neutrino mass is given by

$$
-\mathcal{L}_{\nu}=Y_{\nu_{i j}} \bar{l}_{L i} \tilde{H} N_{j}+\frac{1}{2} M_{N i j} N_{i} N_{j}
$$

where $l_{L i}$ are the left-handed lepton doublets and $M_{N}$ is the Majorona mass matrix of the RH neutrinos. This leads to the light neutrino mass, $m_{\nu}=Y_{\nu}^{T} M_{N}^{-1} Y_{\nu} \frac{v^{2}}{2}$ with $v=246 \mathrm{GeV}$ as the vacuum expectation value of the SM

\footnotetext{
${ }^{2} \mathrm{~A}$ spontaneous breaking of discrete symmetry may lead to cosmological domain wall problem [63]. To circumvent it, one may introduce an explicit $Z_{2}^{\prime}$ breaking term at higher order, which does not affect our analysis.
} 
Higgs. Minimization of the potential $V$ leads to the vevs of $\chi$ and $H^{0}$ (the neutral component of $H$ ), as given by ${ }^{3}$

$$
\begin{aligned}
v_{\chi}^{2} & =6 \frac{2 \mu_{\chi}^{2} \lambda_{H}-\mu_{H}^{2} \lambda_{\chi H}}{2 \lambda_{H} \lambda_{\chi}-3 \lambda_{\chi H}^{2}}, \\
v^{2} & =2 \frac{\mu_{H}^{2} \lambda_{\chi}-3 \mu_{\chi}^{2} \lambda_{\chi H}}{2 \lambda_{H} \lambda_{\chi}-3 \lambda_{\chi H}^{2}} .
\end{aligned}
$$

So, after $\chi$ gets the vev and electroweak symmetry is broken, the mixing between $H^{0}$ and $\chi$ will take place, and new mass or physical eigenstates, $H_{1}$ and $H_{2}$, will be formed. The two physical eigenstates are related with $H^{0}$ and $\chi$ by

$$
\begin{aligned}
& H_{1}=H^{0} \cos \theta-\chi \sin \theta, \\
& H_{2}=H^{0} \sin \theta+\chi \cos \theta,
\end{aligned}
$$

where the mixing angle $\theta$ is defined by

$$
\tan 2 \theta=\frac{\lambda_{\chi H} v v_{\chi}}{-\lambda_{H} v^{2}+\frac{\lambda_{\chi} v_{\chi}^{2}}{6}} .
$$

Similarly, the mass eigenvalues of these physical Higgses are found to be

$$
\begin{aligned}
& m_{H_{1}}^{2}=\frac{\lambda_{\chi}}{6} v_{\chi}^{2}(1-\sec 2 \theta)+\lambda_{H} v^{2}(1+\sec 2 \theta), \\
& m_{H_{2}}^{2}=\frac{\lambda_{\chi}}{6} v_{\chi}^{2}(1+\sec 2 \theta)+\lambda_{H} v^{2}(1-\sec 2 \theta) .
\end{aligned}
$$

Using Eqs. (5)-(7), the couplings $\lambda_{H}, \lambda_{\chi}$, and $\lambda_{\chi H}$ can be expressed in terms of the masses of the physical eigenstates $H_{1}$ and $H_{2}$, the vevs $\left(v, v_{\chi}\right)$, and the mixing angle $\theta$ as

$$
\begin{gathered}
\lambda_{H}=\frac{m_{H_{1}}^{2}}{4 v^{2}}(1+\cos 2 \theta)+\frac{m_{H_{2}}^{2}}{4 v^{2}}(1-\cos 2 \theta), \\
\lambda_{\chi}=\frac{3 m_{H_{1}}^{2}}{2 v_{\chi}^{2}}(1-\cos 2 \theta)+\frac{3 m_{H_{2}}^{2}}{2 v_{\chi}^{2}}(1+\cos 2 \theta), \\
\lambda_{\chi H}=\sin 2 \theta\left(\frac{m_{H_{2}}^{2}-m_{H_{1}}^{2}}{2 v v_{\chi}}\right) .
\end{gathered}
$$

Among $\mathrm{H}_{1}$ and $\mathrm{H}_{2}$, one of them would be the Higgs discovered at the LHC. The other Higgs can be heavier or lighter than the SM Higgs. Below, we proceed to discuss the constraints to be imposed on the couplings and mass parameters of the model before studying the DM phenomenology and vacuum stability in the subsequent sections.

\section{CONSTRAINTS}

Here, we put together the constraints (both theoretical and experimental) that we will take into account to find the parameter space of our model:

(i) To keep the entire potential stable, one needs to maintain the conditions involving the couplings present in $V$ (considering all couplings as real),

$$
\begin{aligned}
\mathbf{S T}_{1,2,3}: & \lambda_{H}>0, \quad \lambda_{\chi}>0, \quad \lambda_{\phi}>0, \\
\mathbf{S T}_{4,5,6}: & \lambda_{\chi H}+\sqrt{\frac{2}{3} \lambda_{H} \lambda_{\chi}}>0, \quad \lambda_{\phi H}+\sqrt{\frac{2}{3} \lambda_{H} \lambda_{\phi}}>0, \quad 3 \lambda_{\chi \phi}+\sqrt{\lambda_{\chi} \lambda_{\phi}}>0, \\
\mathbf{S T}_{7}: & \sqrt{\lambda_{H} \lambda_{\chi} \lambda_{\phi}}+\lambda_{\chi H} \sqrt{\frac{3}{2} \lambda_{\chi}}+3 \lambda_{\phi H} \sqrt{\lambda_{H}}+3 \lambda_{\chi \phi} \sqrt{\lambda_{H}}, \\
& +3\left[\left(\lambda_{\chi H}+\sqrt{\frac{2}{3} \lambda_{H} \lambda_{\chi}}\right)\left(\lambda_{\phi H}+\sqrt{\frac{2}{3} \lambda_{H} \lambda_{\phi}}\right)\left(\lambda_{\chi \phi}+\frac{1}{3} \sqrt{\lambda_{\phi} \lambda_{\chi}}\right)\right]^{1 / 2}>0,
\end{aligned}
$$

which followed from the copositivity of the masssquared matrix involving $H, \chi$, and $\phi$ [66,67].

(ii) In addition, the perturbative unitarity associated with the $S$ matrix corresponding to $2 \rightarrow 2$ scattering processes involving all two-particle initial and final

\footnotetext{
${ }^{3}$ Note that, due to the absence of any $Z_{2}^{\prime}$ breaking term in the Lagrangian of our model, panic vacua $[55,64,65]$ do not appear here.
}

states $[68,69]$ are considered. In the specific model under study, there are 11 neutral and 4 singly charged combinations of two-particle initial/final states. The details are provided in the Appendix. It turns out that the some of the scalar couplings of Eq. (1) are bounded by

$$
\lambda_{H}<4 \pi, \quad \lambda_{\phi H}<8 \pi, \quad \lambda_{\chi H}<8 \pi, \quad \lambda_{\chi \phi}<8 \pi .
$$


The other scalar couplings are restricted (in the form of combinations among them) from the condition that the roots of a polynomial equation should be less than $16 \pi$ [see Eq. (A9)].

(iii) To maintain the perturbativity of all the couplings, we impose the condition that the scalar couplings should remain below $4 \pi$ while Yukawa couplings are less than $\sqrt{4 \pi}$ until $M_{P}$. An upper bound on $\tan \beta\left(=v / v_{\chi}\right)$ follows from the perturbativity of $\lambda_{\chi}$ [70] with a specific choice of $m_{\mathrm{H}_{2}}$.

(iv) Turning into the constraints obtained from experiments, we note that the observed signal strength of the $125 \mathrm{GeV}$ Higgs boson at the LHC [71-76] provides a limit on $\sin \theta$ as $|\sin \theta| \lesssim 0.36$ with $m_{\mathrm{H}_{2}} \gtrsim 150 \mathrm{GeV}$. The analysis in Ref. [77] shows that $\sin \theta$ is restricted significantly $(|\sin \theta| \lesssim 0.3)$ by the direct Higgs searches at colliders [72-76] and combined Higgs signal strength [78] for $150 \mathrm{GeV}<$ $m_{\mathrm{H}_{2}}<300 \mathrm{GeV}$, while for $300 \mathrm{GeV}<m_{\mathrm{H}_{2}}<$ $800 \mathrm{GeV}$, it is the next-to-leading-order contribution to the $W$ boson mass [70] that restricts $\sin \theta$ in a more stipulated range. Corrections to the electroweak precision observables through the $S, T$, and $U$ parameters turn out to be less dominant compared to the limits obtained from $W$ boson mass correction [70]. For our purpose, we consider $\sin \theta \lesssim 0.3$ for the analysis.

Apart from these, we impose the constraints on $Y_{\nu}$ from lepton flavor-violating decays. Also, phenomenological limits obtained on the scalar couplings involved in order to satisfy the relic density $\left(0.1175 \leq \Omega h^{2} \leq 0.1219\right)$ [79] and direct search limits [25] by our dark matter candidate $\phi$ are considered when stability of the EW minimum is investigated.

\section{DARK MATTER PHENOMENOLOGY}

The scalar field $\phi$ playing the role of dark matter has a mass given by $m_{\mathrm{DM}}^{2}=\left(\mu_{\phi}^{2}+\frac{1}{2} \lambda_{\phi H} v^{2}\right)$ as followed from Eq. (1). Before moving toward the relic density calculation in our model, we would like to comment on the simplest $Z_{2}$-odd scalar dark matter scenario in view of the recent XENON 1T [25] result. Note that for the purpose of DM phenomenology in this case the only relevant parameters are given by $m_{\mathrm{DM}}$ and the Higgs portal coupling $\lambda_{\phi H}$ (or $\mu_{\phi}$ and $\lambda_{\phi H}$ ). In Fig. 1 (left panel), we provide a contour plot for relic density consistent with the Planck result [79] in the $\lambda_{\phi H^{-}}-m_{\mathrm{DM}}$ plane indicated by the blue solid line. In the right panel of Fig. 1, we provide the DM-nucleon cross section evaluated with the value of $\lambda_{\phi H}$ corresponding to the $m_{\mathrm{DM}}$ value as obtained from the left panel plot. We then incorporate the direct search limits on the DM-nucleon cross section as obtained from LUX 2016 [24] and the most recent XENON 1T [25] result [25] in the same plot denoted by blue and red dashed lines, respectively. We conclude that the dark matter mass below $500 \mathrm{GeV}$ is excluded from the present XENON 1T [25] result. This result is indicated by the red portion of the contour line in the left panel, while the remaining blue portion of the contour plot (of the left panel) represents the allowed range of $m_{\mathrm{DM}}$ satisfying both the relic density and direct search constraints.

Let us now move to the relic density estimate in our setup with the extra scalar $\chi$ and compare the phenomenology with the simplest scalar DM scenario in the light of the mixing between the SM Higgs and $\chi$. Using Eq. (4) and inserting them into the SM Lagrangian along with the ones mentioned in Eq. (1), we obtain the following list of interaction vertices involving two Higgses $\left(H_{1}\right.$ and $\left.H_{2}\right)$, the dark matter field $(\phi)$, and several other SM fields:
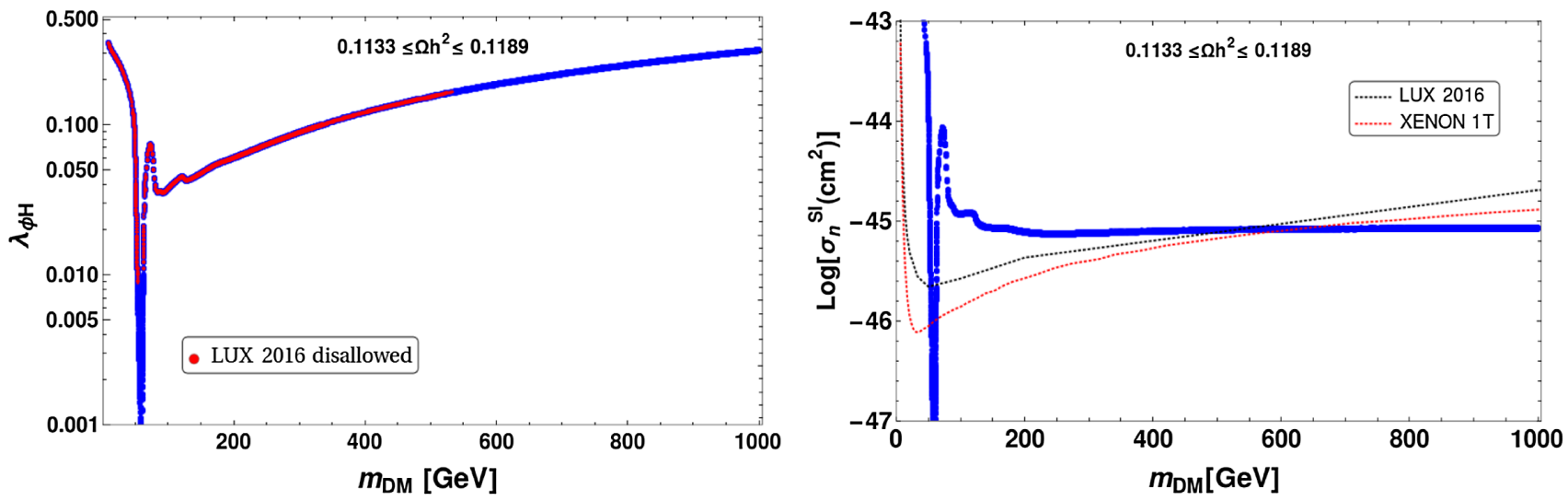

FIG. 1. (Left panel) Relic density contour plot in the $m_{\mathrm{DM}}-\lambda_{\phi H}$ plane; the red portion corresponds to the disfavored range of parameters by recent direct detection results, while the blue portion stands for the allowed region of parameters consistent with direct detection results. (Right panel) The spin-independent cross section is plotted (blue line) for relic density allowed points as a function of $m_{\mathrm{DM}}$, where the LUX 2016 and XENON 1T limits are indicated by blue and red dashed lines, respectively. 


$$
\begin{aligned}
H_{1} f \bar{f}, H_{2} f \bar{f}: & \frac{m_{f}}{v} \cos \theta, \frac{m_{f}}{v} \sin \theta \\
H_{1} Z Z, H_{2} Z Z: & \frac{2 m_{Z}^{2}}{v} \cos \theta g^{\mu \nu}, \frac{2 m_{Z}^{2}}{v} \sin \theta g^{\mu \nu} \\
H_{1} W^{+} W^{-}, H_{2} W^{+} W^{-}: & \frac{2 m_{W}^{2}}{v} \cos \theta g^{\mu \nu}, \frac{2 m_{W}^{2}}{v} \sin \theta g^{\mu \nu} \\
\phi \phi H_{1}: & -v_{\chi} \lambda_{\chi \phi} \sin \theta+v \lambda_{\phi H} \cos \theta \equiv \lambda_{1} \\
\phi \phi H_{2}: & v_{\chi} \lambda_{\chi \phi} \cos \theta+v \lambda_{\phi H} \sin \theta \equiv \lambda_{2} \\
\phi \phi H_{1} H_{1}: & \lambda_{\phi H} \cos ^{2} \theta+\lambda_{\chi \phi} \sin ^{2} \theta \\
\phi \phi H_{2} H_{2}: & \lambda_{\phi H} \sin ^{2} \theta+\lambda_{\chi \phi} \cos ^{2} \theta \\
\phi \phi H_{1} H_{2}: & \left(\lambda_{\phi H}-\lambda_{\chi \phi}\right) \sin \theta \cos \theta \\
H_{1} H_{1} H_{1}: & {\left[6 v \lambda_{H} \cos { }^{3} \theta-3 v_{\chi} \lambda_{\chi H} \cos { }^{2} \theta \sin \theta+3 v \lambda_{\chi H} \cos \theta \sin ^{2} \theta-v_{\chi} \lambda_{\chi} \sin ^{3} \theta\right] } \\
H_{2} H_{2} H_{2}: & {\left[6 v \lambda_{H} \sin ^{3} \theta+3 v_{\chi} \lambda_{\chi H} \cos \theta \sin ^{2} \theta+3 v \lambda_{\chi H} \cos { }^{2} \theta \sin \theta+v_{\chi} \lambda_{\chi} \cos ^{3} \theta\right] } \\
H_{1} H_{1} H_{2}: & {\left[2 v\left(3 \lambda_{H}-\lambda_{\chi H}\right) \cos ^{2} \theta \sin \theta+v \lambda_{\chi H} \sin ^{3} \theta+v_{\chi}\left(\lambda_{\chi}-2 \lambda_{\chi H}\right) \cos ^{2} \theta \sin ^{2} \theta+v_{\chi} \lambda_{\chi H} \cos ^{3} \theta\right] } \\
H_{1} H_{2} H_{2}: & {\left[2 v\left(3 \lambda_{H}-\lambda_{\chi H}\right) \cos ^{2} \theta \sin ^{2} \theta+v \lambda_{\chi H} \cos ^{3} \theta-v_{\chi}\left(\lambda_{\chi}-2 \lambda_{\chi H}\right) \cos ^{2} \theta \sin ^{2} \theta-v_{\chi} \lambda_{\chi H} \sin ^{3} \theta\right] . }
\end{aligned}
$$

Following Eq. (13), we draw the Feynman diagrams for DM annihilation channels into SM particles and to the second Higgs in Fig. 2.

It is expected that the DM candidate is in thermal equilibrium with the SM degrees of freedom in the early Universe. We therefore proceed to evaluate their abundance through the standard freeze-out mechanism. The Boltzmann equation,

$$
\dot{n}_{\phi}+3 \mathrm{H} n_{\phi}=-\left\langle\sigma v_{\phi \phi}\right\rangle\left(n_{\phi}^{2}-n_{\phi}^{\mathrm{eq} 2}\right),
$$

is employed for this purpose, where $n_{\phi}$ is the number density of the dark matter $\phi, \mathrm{H}$ is the Hubble parameter, and $\left\langle\sigma v_{\phi \phi}\right\rangle$ represents the total annihilation cross section as given by $\left\langle\sigma v_{\phi \phi}\right\rangle=\left\langle\sigma v_{\phi \phi \rightarrow \mathrm{SM}, \mathrm{SM}}\right\rangle+\left\langle\sigma v_{\phi \phi \rightarrow H_{1} H_{2}}\right\rangle+$ $\left\langle\sigma v_{\phi \phi \rightarrow H_{2} H_{2}}\right\rangle$. We consider here the RH neutrinos to be massive enough compared to the DM mass. So, RH neutrinos do not participate in DM phenomenology. We have then used the MicROMEGA package [80] to evaluate the final relic abundance of DM.

We have the following parameters in our setup:

$$
\left\{m_{H_{1}}, m_{H_{2}}, m_{\mathrm{DM}}, \sin \theta, \lambda_{\chi \phi}, \lambda_{\phi H}, v, \tan \beta, \lambda_{\phi}\right\} .
$$

The parameter $v_{\chi}$ is involved in the definition of $\tan \beta=v / v_{\chi}$. Parameters $\left(\lambda_{H}, \lambda_{\chi}, \lambda_{\chi H}\right)$ can be written in terms of other parameters as shown in Eqs. (8)-(10).

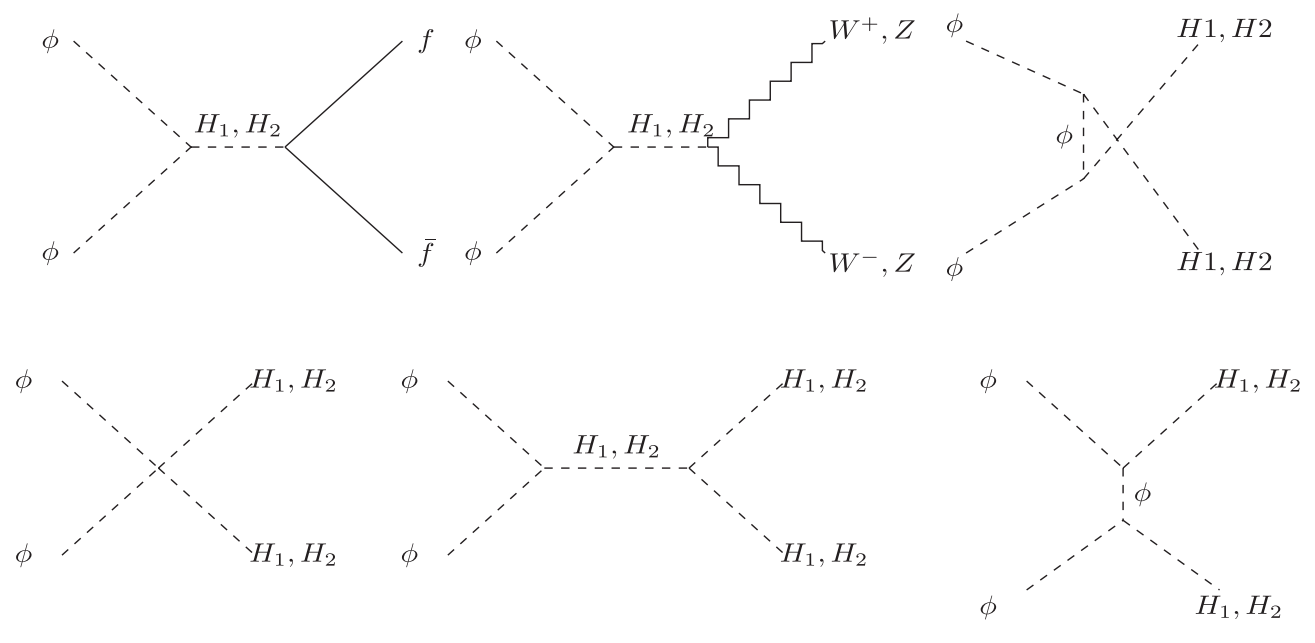

FIG. 2. Diagrams contributing to $\phi \phi$ annihilation to SM particles and the other Higgs. 
Among all the parameters in Eq. (15), $\lambda_{\phi}$ does not play any significant role in DM analysis.

We first assume $H_{1}$ as the Higgs discovered at the LHC, i.e, $m_{H_{1}} \sim 125.09 \mathrm{GeV}$ [4], and the other Higgs is the heavier one $\left(m_{H_{2}}>m_{H_{1}}\right)$. It would be appealing in view of the LHC accessibility to keep $m_{H_{2}}$ below $1 \mathrm{TeV}$. In this case, limits on $\sin \theta, \tan \beta$ are applicable as discussed in Sec. III depending on specific value of $m_{\mathrm{H}_{2}}$ [77]. Now, in this regime (where $m_{H_{2}}$ is not too heavy, in particular, $m_{H_{2}}<1 \mathrm{TeV}$ ), $\sin \theta$ is bounded by $\sin \theta \lesssim 0.3$ [77], and we have taken here a conservative choice by fixing $\sin \theta=0.2$. Note that in the small $\sin \theta$ approximation $H_{1}$ is mostly dominated by the SM Higgs doublet $H$. In this limit, the second term in Eq. (8) effectively provides the threshold correction to $\lambda_{H}[56,81,82]$, which helps achieve vacuum stability as we will see later. Furthermore, considering this threshold effect to be equal to or less than the first term in Eq. (8) (i.e., approximately the SM value of $\lambda_{H}$ ), we obtain an upper bound on $m_{H_{2}}$ as $m_{H_{2}}<\frac{m_{H_{1}}}{\tan \theta}$. Therefore, in case with $m_{H_{2}}>m_{H_{1}}$, our working regime of $m_{H_{2}}$ can be considered within $\frac{m_{H_{1}}}{\tan \theta}>m_{H_{2}}>m_{H_{1}}$. We take $m_{H_{2}}$ to be $300 \mathrm{GeV}$ for our analysis.

Note that with small $\theta, \lambda_{\chi}$ almost coincides with the second term in Eq. (9). It is quite natural to keep the magnitude of a coupling below unity to maintain the perturbativity limit for all energy scales including its running. Hence, with the demand $\lambda_{\chi}<1$, one finds $v_{\chi}>\sqrt{3} m_{\mathrm{H}_{2}}$. To show it numerically, let us choose $\sin \theta=0.2$; then, we obtain $125 \mathrm{GeV}<$ $m_{H_{2}}<620 \mathrm{GeV}$. Therefore, with $m_{H_{2}}=300 \mathrm{GeV}$, a lower limit on $v_{\chi} \geq 520 \mathrm{GeV}$ can be set. We consider $v_{\chi}$ to be $800 \mathrm{GeV}$ so that $\tan \beta$ turns out to be 0.307 .

On the other hand, if we consider the other Higgs to be lighter than the one discovered at LHC, we identify $m_{H_{2}}$ to be the one found at the LHC and hence $m_{H_{1}} \leq 125 \mathrm{GeV}$. Then, Eq. (4) suggests $\sin \theta \rightarrow 1$ as the complete decoupling limit of the second Higgs. Following the analysis in Refs. [77,83-87], we infer that most of the parameter space except for a very narrow region both in terms of mixing angle $(\sin \theta \sim 0.9)$ and mass of the lighter Higgs $\left(m_{H_{1}} \sim 85-100 \mathrm{GeV}\right)$ is excluded from Large ElectronPositron Collider (LEP) and LHC searches. Such a range is not suitable for our purpose, as can bee seen from Eq. (8). In this large $\sin \theta$ limit, $\lambda_{H}$ gets the dominant contribution from the second term in Eq. (8), in which the first term serves the purpose of threshold effect on $\lambda_{H}$. However, $m_{H_{1}}$ being smaller than $m_{H_{2}}$ (the SM like Higgs), this effect would not be sufficient to enhance $\lambda_{H}$ such that its positivity until $M_{P}$ can be ensured. Therefore, we discard the scenario $m_{H_{1}}<m_{H_{2}}$ (SM-like Higgs) from our discussion. Hence, the DM phenomenology basically depends on $m_{\mathrm{DM}}, \sin \theta, \lambda_{\chi \phi}$, and $\lambda_{\phi H}$.

In a direct detection experiment, the DM scatters with the nucleon through the exchange of $H_{1}$ and $H_{2}$ as shown

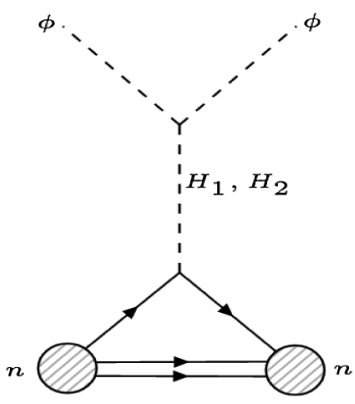

FIG. 3. Feynman diagram for DM direct detection.

schematically in Fig. 3. The resulting spin-independent cross section of DM-nucleon elastic scattering is given by Ref. [35],

$$
\sigma_{n}^{\mathrm{SI}}=\frac{f_{n}^{2} \mu_{n}^{2} m_{n}^{2}}{4 \pi v^{2} m_{\mathrm{DM}}^{2}}\left[\frac{\lambda_{1} \cos \theta}{m_{H_{1}}^{2}}+\frac{\lambda_{2} \sin \theta}{m_{H_{2}}^{2}}\right]^{2},
$$

where $\mu_{n}=\frac{m_{n} m_{\mathrm{DM}}}{m_{n}+m_{\mathrm{DM}}}$ and $f_{n}=0.284$ [88,89]. The couplings appeared as $\lambda_{1}$ and $\lambda_{2}$ are specified in the list of vertices in Eq. (13). Below, we discuss how we can estimate the relevant parameters $\left(\lambda_{\phi_{H}}, \lambda_{\chi \phi}\right.$, and $\left.m_{\mathrm{DM}}\right)$ from relic density and direct search limits. For this purpose, we consider $m_{H_{2}}=300 \mathrm{GeV}$ and $v_{\chi}=800 \mathrm{GeV}$ as reference values, unless otherwise mentioned.

\section{A. DM mass in region $\mathrm{R} 1: 150 \mathrm{GeV}<\boldsymbol{m}_{\mathrm{DM}} \leq 500 \mathrm{GeV}$}

In this region, any decay mode of $H_{1}$ and $H_{2}$ into DM is kinematically forbidden, following our consideration for $m_{H_{2}}=300 \mathrm{GeV}$. As stated before, we consider $m_{H_{1}}$ to be the SM-like Higgs discovered at the LHC, with $v_{\chi}=$ $800 \mathrm{GeV}$ and $\tan \beta$ being fixed at 0.307 . Therefore, to satisfy the relic density $\Omega h^{2}=0.1161 \pm 0.0028$ [79], we first scan over $\lambda_{\phi H}$ and $\lambda_{\chi \phi}$ for different ranges of dark matter mass where $\sin \theta$ is kept fixed at 0.2. The allowed range of parameter space contributing to the relic abundance satisfying the correct relic density is indicated on the $\lambda_{\phi H^{-}}-\lambda_{\chi \phi}$ plane in Fig. 4 (in the top-left panel), where different colored patches indicate different ranges of $m_{\mathrm{DM}}$. In the upper-right plot of Fig. 4, the corresponding direct search cross sections for the relic density satisfying points obtained from the upper-left plot (including the variation of $\lambda_{\phi H}$ and $\lambda_{\chi \phi}$ ) are provided. It can be clearly seen that many of these points lie below the LUX 2016 [24] experimental limit for a wide range of dark matter mass (indicated by the colors depicted in the inset of Fig. 4, upper-left panel).

From the top-left panel of Fig. 4, the relic density contour plot (with a particular $m_{\mathrm{DM}}$ ) in the $\lambda_{\chi \phi}-\lambda_{\phi H}$ plane shows that there exists a range of $\lambda_{\phi H}$ for which the plot is (almost) insensitive to the change in $\lambda_{\chi \phi}$. This becomes more prominent for plots associated with higher dark matter mass. In particular, the contour line satisfying the 

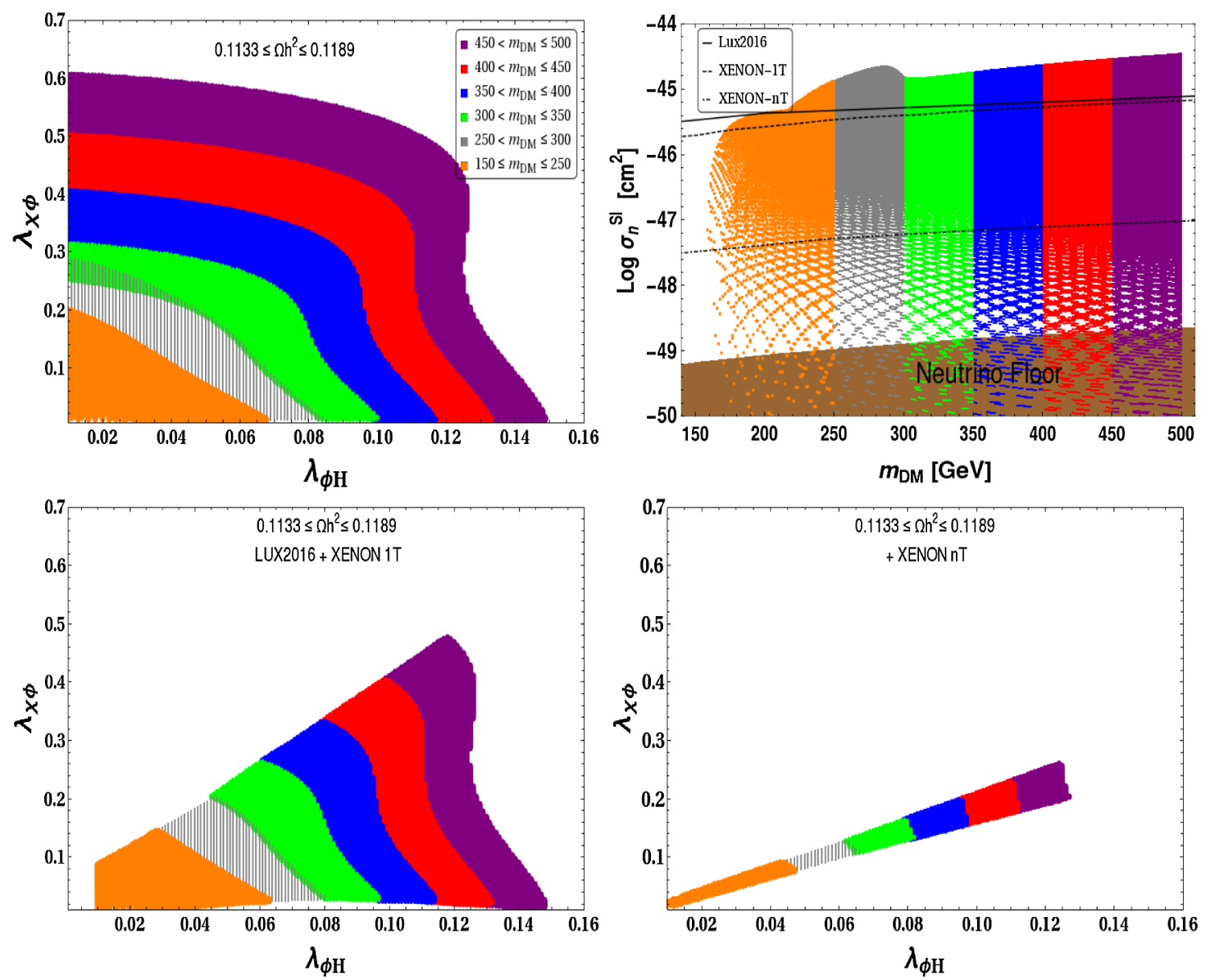

FIG. 4. (Top left) Allowed points on the $\lambda_{\phi H}-\lambda_{\chi \phi}$ plane for DM having mass $150<m_{\mathrm{DM}}<500$ GeV to satisfy the correct order of relic density. (Top right) The spin-independent nucleon cross section of DM has been plotted against the DM mass. (Bottom panel) The top-left plot has been constrained using recent LUX 2016 [24] and Xenon 1T [25] limits to produce the bottom-left figure and Xenon nT [27] predictions to get the bottom-right figure.

correct relic density with $m_{\mathrm{DM}}=500 \mathrm{GeV}$ depicts a sharp variation in $\lambda_{\chi \phi}$ (below 0.4) with almost no variation of $\lambda_{\phi H}$ around 0.13 . We now discuss the reason behind such a behavior. We note that for $\lambda_{\phi H}>0.13$ the total annihilation cross section satisfying the relic density is mostly dominated by the $\phi \phi \rightarrow \mathrm{SM}$, SM process, specifically, $\phi \phi \rightarrow W W, Z Z$ dominate. In our scenario, $\phi \phi \rightarrow W W, Z Z$ processes are mediated by both the Higgses, $H_{1}$ and $H_{2}$. Although $\lambda_{\chi \phi}$ is involved in the vertices characterizing these processes, it turns out that once both the $H_{1}$ and $H_{2}$ contributions are taken into account the $\lambda_{\chi \phi}$ dependence is effectively canceled, leaving the $\phi \phi \rightarrow W W, Z Z$ annihilation almost independent of $\lambda_{\chi \phi}$. Hence, $\phi \phi \rightarrow \mathrm{SM}$, SM depends mostly on $\lambda_{\phi H}$. The other processes like $\phi \phi \rightarrow$ $\mathrm{H}_{1} \mathrm{H}_{2}\left(\mathrm{H}_{2} \mathrm{H}_{2}\right)$ are subdominant [these are allowed, provided $\left.m_{\mathrm{DM}}>212.5(300) \mathrm{GeV}\right]$ in this region with large $\lambda_{\phi H}$. Then, the total cross section $\left\langle\sigma v_{\phi \phi}\right\rangle$ and hence the relic density contour line become insensitive to the change in $\lambda_{\chi \phi}$ as long as it remains below 0.4 while $\lambda_{\phi H}>0.13$. This is evident in the top-left panel of Fig. 4. Similar effects are seen in the case of lower $m_{\mathrm{DM}}(<500 \mathrm{GeV})$ as well.
Once we keep decreasing $\lambda_{\phi H}$ below 0.13 , it turns out that $\phi \phi \rightarrow$ SM, SM becomes less important compared to $\phi \phi \rightarrow$ $\mathrm{H}_{2} \mathrm{H}_{2}$ (in particular, the $t$ channel) with $\lambda_{\chi \phi}$ beyond 0.4 (in the case of $m_{\mathrm{DM}}=500 \mathrm{GeV}$ ). Note that the plot shows the insensitivity related to $\lambda_{\phi H}$ in this low- $\lambda_{\phi H}$ region for obvious reasons. Similar results follow with $m_{\mathrm{DM}}<300 \mathrm{GeV}$ also, where $\phi \phi \rightarrow H_{1} H_{2}$ provides the dominant contribution in $\left\langle\sigma v_{\phi \phi}\right\rangle$. Based on our discussion so far, we note that for $\lambda_{\chi \phi} \gg \lambda_{\phi H}$ the channels with Higgses in the final states contribute more to total $\left\langle\sigma v_{\phi \phi}\right\rangle$. On the other hand, for low values of $\lambda_{\chi \phi}$ (although comparable to $\lambda_{\phi H}$ ), the model resembles the usual Higgs portal dark matter scenario in which $\mathrm{W}$ bosons in the final state dominate. To summarize:

(i) $150 \mathrm{GeV}<m_{\mathrm{DM}}<212.5 \mathrm{GeV}$ : For low $\lambda_{\chi \phi}, \phi \phi \rightarrow$ $W^{+} W^{-}$dominates. However, for large $\lambda_{\chi \phi}, \phi \phi \rightarrow$ $H_{1} H_{1}$ becomes the main annihilation channel.

(ii) $212.5 \mathrm{GeV}<m_{\mathrm{DM}}<300 \mathrm{GeV}$ : The new annihilation process $\phi \phi \rightarrow H_{1} H_{2}$ opens up. This with $\phi \phi \rightarrow H_{1} H_{1}$ contributes dominantly for large $\lambda_{\chi \phi}$. Otherwise, the channels with SM particles in final states dominate. 
(iii) $300 \mathrm{GeV}<m_{\mathrm{DM}}<500 \mathrm{GeV}$ : The annihilation channel $\phi \phi \rightarrow \mathrm{H}_{2} \mathrm{H}_{2}$ opens up in addition to $H_{1} H_{1}$ and $H_{1} H_{2}$ in the final states. Their relative contributions to total $\left\langle\sigma v_{\phi \phi}\right\rangle$ again depend on the value of $\lambda_{x \phi}$.

In the top-left panel of Fig. 4, we also note the existence of a small overlapped region when $\lambda_{\phi H} \ll \lambda_{x \phi}$ for the dark matter mass regions between 280 and $300 \mathrm{GeV}$ and 300 and $310 \mathrm{GeV}$. This has been further clarified in Fig. 5, where we note that relic density contour lines with $m_{\mathrm{DM}}=299 \mathrm{GeV}$ and $m_{\mathrm{DM}}=305 \mathrm{GeV}$ intersect each other around $\lambda_{\phi H} \sim 0.05$ and $\lambda_{\chi \phi} \sim 0.21$. Note that when DM mass $m_{\mathrm{DM}} \geq m_{H_{2}}=300 \mathrm{GeV}$, in addition to the $\phi \phi \rightarrow \mathrm{SM}$, SM and $\phi \phi \rightarrow H_{1} H_{2}$ annihilation processes, $\phi \phi \rightarrow$ $\mathrm{H}_{2} \mathrm{H}_{2}$ opens up and contributes to the total annihilation cross section (this new channel can be realized through both $H_{1}$ and $H_{2}$ mediation).

Then, the total annihilation cross section will be enhanced for the $m_{\mathrm{DM}}>300 \mathrm{GeV}$ case; i.e., $\left\langle\sigma v_{\phi \phi}\right\rangle=$ $\langle\sigma v\rangle_{\phi \phi \rightarrow \mathrm{SM}, \mathrm{SM}}+\langle\sigma v\rangle_{\phi \phi \rightarrow H_{1}, H_{2}}+\langle\sigma v\rangle_{\phi \phi \rightarrow H_{2}, H_{2}}$ becomes large compared to the $280 \mathrm{GeV}<m_{\mathrm{DM}}<300 \mathrm{GeV}$ mass range in which $\langle\sigma v\rangle_{\phi \phi \rightarrow H_{2} H_{2}}$ is not present. This enhancement has to be nullified in order to realize the correct relic density, and this is achieved by reducing $\lambda_{\chi \phi}$ compared to its required value for a fixed $\lambda_{\phi H}$ and $m_{\mathrm{DM}}$ in the $280 \mathrm{GeV} \leq m_{\mathrm{DM}}<300$ region. Note that in view of our previous discussion we already understand that $\phi \phi \rightarrow$ $\mathrm{H}_{2} \mathrm{H}_{2}$ becomes important compared to the $\phi \phi \rightarrow \mathrm{SM}$, SM process in the region with $\lambda_{\chi \phi} \gg \lambda_{\phi H}$. Hence, the two mass regions (below and above $300 \mathrm{GeV}$ ) overlap in $\lambda_{\phi H}-\lambda_{\chi \phi}$ plane as seen in the top-left panel of Fig. 4 as well as in Fig. 5. The total annihilation cross section of DM depends on its mass also. However, the small mass differences between the two overlapped regions have very a mild effect on $\langle\sigma v\rangle_{\text {Tot }}$. A similar effect should be observed

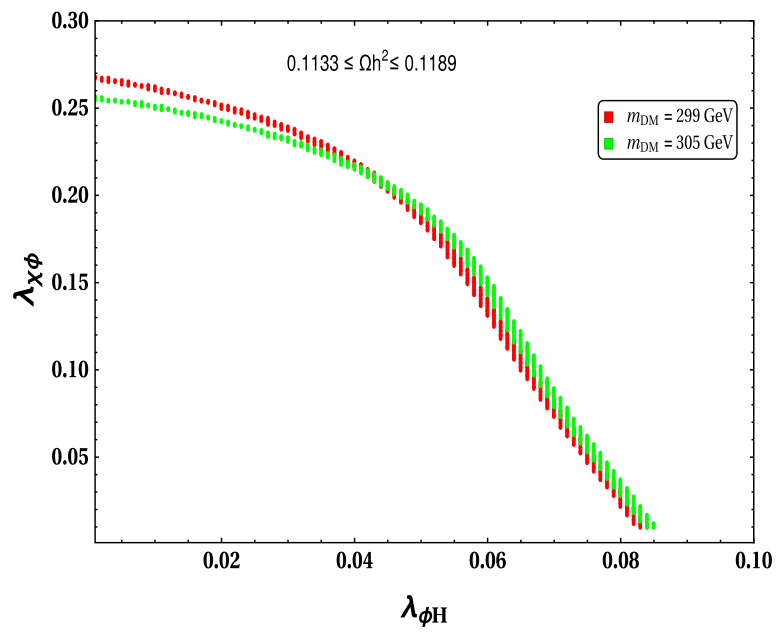

FIG. 5. DM relic density contour lines in the $\lambda_{\phi H}-\lambda_{\chi \phi}$ plane with $m_{\mathrm{DM}}=299$ (red) and $305 \mathrm{GeV}$ (green). below and above $m_{\mathrm{DM}} \sim\left(m_{H_{1}}+m_{H_{2}}\right) / 2=212.5 \mathrm{GeV}$ as $\phi \phi \rightarrow H_{1} H_{2}$ opens up there. However, we find that around the $m_{\mathrm{DM}}=212.5 \mathrm{GeV}$, even with $\lambda_{\chi \phi} \gg \lambda_{\phi H}$, the contribution from this particular channel to $\langle\sigma v\rangle_{\text {Tot }}$ is negligible as compared to the $\phi \phi \rightarrow \mathrm{SM}, \mathrm{SM}$ contribution, and hence we do not observe any such overlapped region there.

In the top-right panel of Fig. 4, we provide the spinindependent (SI) direct detection (DD) cross sections corresponding to the points in the left panel satisfying relic density data having different ranges of dark matter masses as indicated by the colored patches. We further put the LUX 2016 [24], XENON 1T [25], and nT (expected) lines on it. As known, for a lower cross section, it reaches the neutrino floor where signals from DM cannot be distinguished from those of a neutrino. We find that the scenario works with reasonable values of the parameters, i.e., not with any unnaturally small or large values of couplings. Note that once we use the XENON 1T [25] and projected XENON nT [27] limits on the scattering cross section we will obtain a more restricted region of parameter space for $\lambda_{\phi H}-\lambda_{\chi \phi}$ as shown in the left (with XENON 1T [25]) and right (with XENON nT [27]) figures of the bottom panel. From the plot with XENON-nT prediction, we find that the scenario works even with reasonably large values of $\lambda_{\phi H}$ and $\lambda_{\chi \phi}$ required to satisfy the relic density, although they are comparable to each other. This is because, to keep the direct detection cross section relatively small (even smaller than the XENON nT), it requires a cancellation between $\lambda_{\phi H}$ and $\lambda_{\chi \phi}$, as can be seen from Eq. (16) in conjugation with definition of $\lambda_{1}$ and $\lambda_{2}$ for a specific $\sin \theta=0.2$ value. Such a cancellation is not that important for plots with LUX 2016 [24] or XENON 1T [25] results and hence show a wider region of parameter space for $\lambda_{\chi \phi}$ and $\lambda_{\phi H}$.

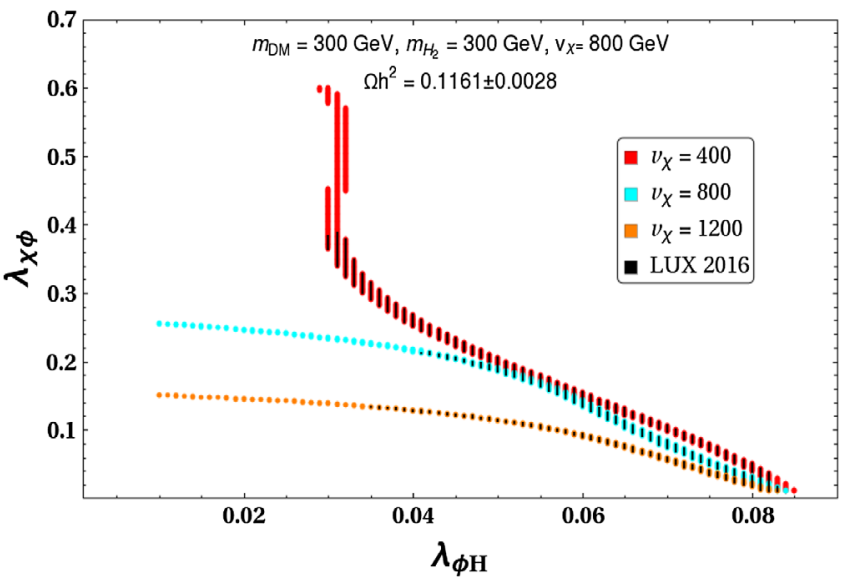

FIG. 6. Allowed parameter space to satisfy correct relic abundance in the $\lambda_{\phi H}-\lambda_{\chi \phi}$ plane with different $v_{\chi}$ for $m_{\mathrm{DM}}=300 \mathrm{GeV}$. Other parameters $m_{\mathrm{H}_{2}}=300 \mathrm{GeV}$ and $\sin \theta=0.2$ have been kept fixed. The LUX 2016 [24] allowed regions are also accommodated (solid black region) in the figures. 

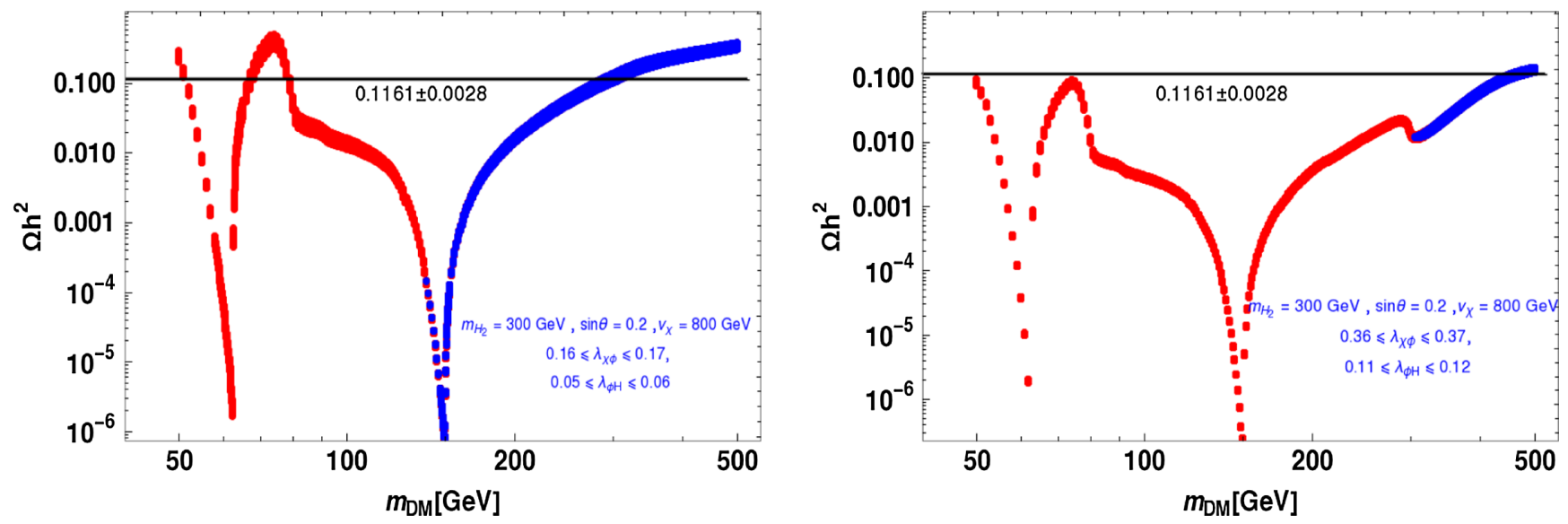

FIG. 7. Relic density vs the $m_{\mathrm{DM}}$ plot in the combined setup of SM $+\mathrm{DM}+\mathrm{RH}$ neutrinos and the $\chi$ field for two different specified ranges of $\lambda_{\phi H}$ and $\lambda_{\chi \phi}$ as mentioned within the inset of the figures. Two resonances are clearly visible at $m_{\mathrm{DM}}=m_{H_{1}} / 2$ and $m_{H_{2}} / 2$. The blue patch represents the favored region by the LUX 2016 direct detection cross section limit, whereas the red patch is excluded by LUX 2016.

It can be concluded from upper panel of Fig. 4 that the presence of an additional singlet scalar field $\chi$ helps reduce the magnitude of $\lambda_{\phi H}$ that was required (say $\lambda_{\phi H}^{0}$ ) to produce correct relic density in the minimal form of singlet scalar DM, or in other words, it dilutes the pressure on $\lambda_{\phi H}$ to produce the correct relic density and to satisfy DD cross section simultaneously. For illustrative purposes, let us choose a dark matter mass with $500 \mathrm{GeV}$. From Fig. 1, we find that in order to satisfy the relic density we need to have $\lambda_{\phi H}^{0} \sim, 0.15$ which can even be 0.02 in the case with large $\lambda_{\chi \phi} \sim 0.6$. Similarly, we notice that for $m_{\mathrm{DM}}=300 \mathrm{GeV}$ $\lambda_{\phi H}^{0}$ was 0.086 in order to produce the correct relic density, which, however, was excluded from a direct search point of view. This conclusion changes in the presence of $\lambda_{\chi \phi}$, as we can see from Fig. 4 (left panel) that $m_{\mathrm{DM}}=300 \mathrm{GeV}$ can produce the correct relic density and evade the direct search limit with smaller $\lambda_{\phi H}: 0.065-0.086$. This is possible in the presence of nonzero $\lambda_{\chi H}$ and small $\sin \theta(\sim 0.2$ here $)$, which redistribute the previously obtained value of $\lambda_{\phi H}^{0}$ into $\lambda_{\phi H}$ and $\lambda_{\chi \phi}$ while simultaneously bringing the direct search cross section to be less than the experimental limit due to its association with $\sin \theta$ (see the definitions of $\lambda_{1}$ and $\lambda_{1}$ ).

In Fig. 7 (left panel), we show the relic density vs the $m_{\mathrm{DM}}$ plot with our chosen set of parameters, $\left\{m_{\mathrm{H}_{2}}=\right.$ $\left.300 \mathrm{GeV}, m_{H_{1}}=125.09 \mathrm{GeV}, \tan \beta=0.307, \sin \theta=0.2\right\}$, while varying $\lambda_{\chi H}$ and $\lambda_{\phi H}$ within $0.16 \leq \lambda_{\chi \phi} \leq 0.17$ and $0.05 \leq \lambda_{\phi H} \leq 0.06$. Similarly, in the right panel, we provide the relic density vs the $m_{\mathrm{DM}}$ plot for a different range of $\lambda_{\chi H}$ and $\lambda_{\phi H}$. We note that there are two resonance regions, one at $m_{H_{1}} / 2$ for the SM-like Higgs and another at $m_{\mathrm{H}_{2}} / 2$ with a heavy Higgs ${ }^{4}$ mass at $300 \mathrm{GeV}$. In left panel

\footnotetext{
${ }^{4}$ As expected, it would be always possible to satisfy the relic density and DD limits within this region.
}

for DM heavier than $150 \mathrm{GeV}$, we find $m_{\mathrm{DM}} \sim 300 \mathrm{GeV}$ can correctly produce the relic density in the observed range and simultaneously evade the DD limit set by LUX 2016 [24]. This result is consistent with the plot in Fig. 4. Similarly, $m_{\mathrm{DM}} \sim 500 \mathrm{GeV}$ is in the acceptable range, which is in line with observation in Fig. 4. In the left panel of Fig. 7, we also have another region of DM mass $75 \mathrm{GeV}$ having the correct relic abundance, however, discarded by LUX 2016. The region was not incorporated in top-left panel of Fig. 4, as we have started with $m_{\mathrm{DM}}$ bigger than $150 \mathrm{GeV}$ only. The possibility of having dark matter lighter than $150 \mathrm{GeV}$ in the present scenario will be discussed in the next subsection. Since, in obtaining the Fig. 4, we have fixed $\sin \theta, \tan \beta$, and $m_{\mathrm{H}_{2}}$, below in Figs. 6 and 8 , we provide the expected range of two couplings $\lambda_{\chi H}$ and $\lambda_{\phi H}$ when $\sin \theta$ and $\tan \beta$ are varied for dark matter mass $m_{\mathrm{DM}}=300 \mathrm{GeV}$. We find only a little sensitive with the change of both $v_{\chi}$ and $\sin \theta$. As $v_{\chi}$ or $\sin \theta$ increases for $m_{\mathrm{DM}}=300 \mathrm{GeV}$, it requires less $\lambda_{\chi \phi}$ for a particular $\lambda_{\phi H}$ to satisfy the relic density. We have also applied the LUX 2016 [24] DD cross section limit in those plots, which are indicated by solid black patches. In Fig. 8, one dark blue dot has been put on the $\sin \theta=0.2$ contour, which will be used in the study of Higgs vacuum stability as a reference point.

\section{B. DM mass in region R2: $\boldsymbol{m}_{\mathrm{DM}}<150 \mathrm{GeV}$}

Here, we briefly discuss the DM phenomenology in the low-mass region $m_{\mathrm{DM}}<\frac{m_{\mathrm{H}_{2}}}{2}=150 \mathrm{GeV}$. In this region, the decay process of heavy Higgs to DM $\left(H_{2} \rightarrow \phi \phi\right)$ will be active. For further low $m_{\mathrm{DM}}<m_{H_{1}} / 2 \simeq 62.5 \mathrm{GeV}$, both $H_{2} \rightarrow \phi \phi$ and $H_{1} \rightarrow \phi \phi$ decay modes will be present.

We perform a scan over the $\lambda_{\phi H}-\lambda_{\chi \phi}$ region to find the correct relic density satisfied parameter space with the allowed direct detection cross section from LUX 2016 [24] 


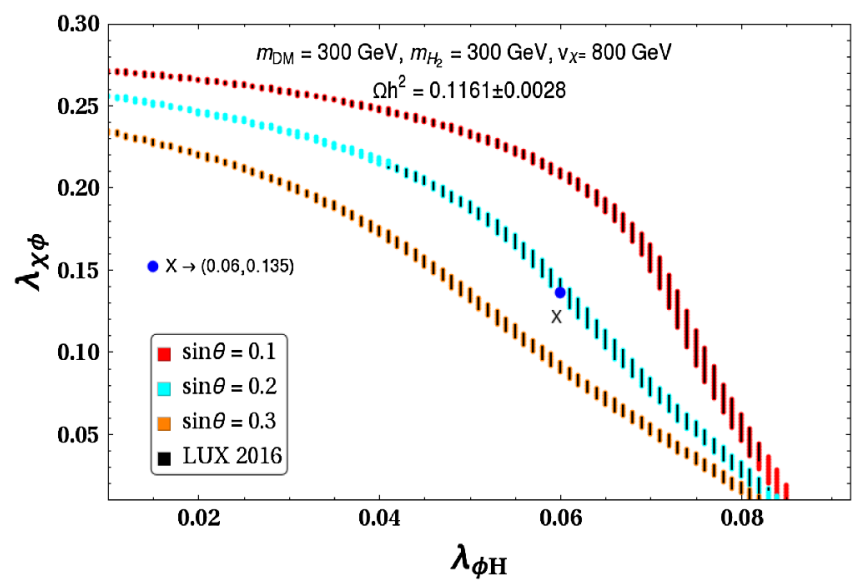

FIG. 8. Allowed parameter space to satisfy correct relic abundance in the $\lambda_{\phi H^{-}}-\lambda_{\chi \phi}$ plane with different values of $\sin \theta$ for $m_{\mathrm{DM}}=300 \mathrm{GeV}$. Other parameters $m_{\mathrm{H}_{2}}=300 \mathrm{GeV}$ and $v_{\chi}=800 \mathrm{GeV}$ have been kept fixed. The LUX 2016 [24] allowed region is also accommodated (solid black region) in the figures. The blue dot denoted by $\mathrm{X}$ in the right panel will be used as a reference point for the study on Higgs vacuum stability.

and XENON 1T experiments [25]. The results are shown in Fig. 9, left and right panels, in which DD limits from LUX 2016 and XENON 1T (preliminary), respectively, are considered separately. In doing these plots, we have considered different mass ranges, as indicated by different colors. The color codes are depicted within the inset of each figure. We note that the required $\lambda_{\chi \phi}$ and $\lambda_{\phi H}$ values are almost in the range similar to that obtained in Fig. 4. We also note that there exists a resonance region through $H_{1}$ near $m_{\mathrm{DM}} \sim 63 \mathrm{GeV}$, indicated by the blue patch. In this resonance region, the relic density becomes insensitive to the coupling, and hence the blue patch is extended over the entire region of $\lambda_{\chi \phi}$ and $\lambda_{\phi H}$ in Fig. 9.

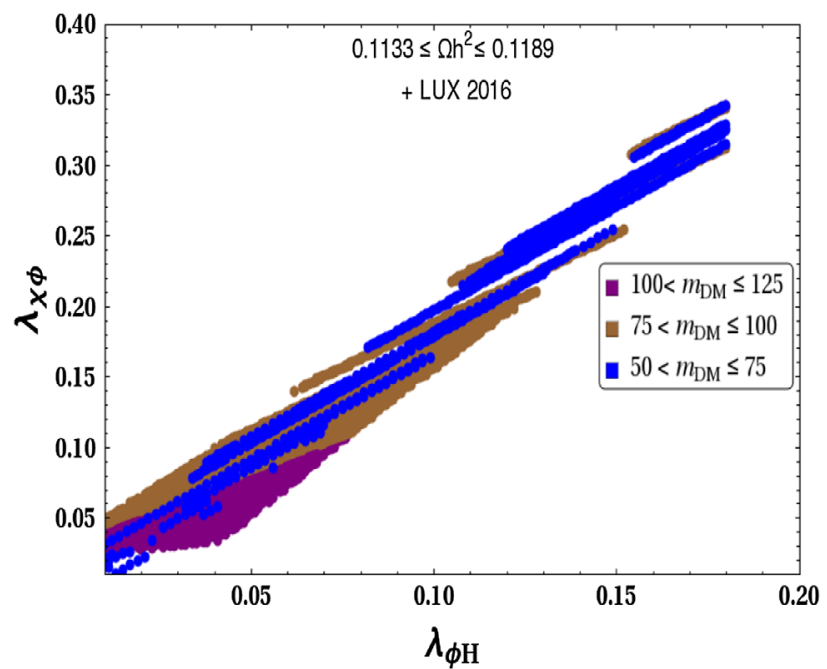

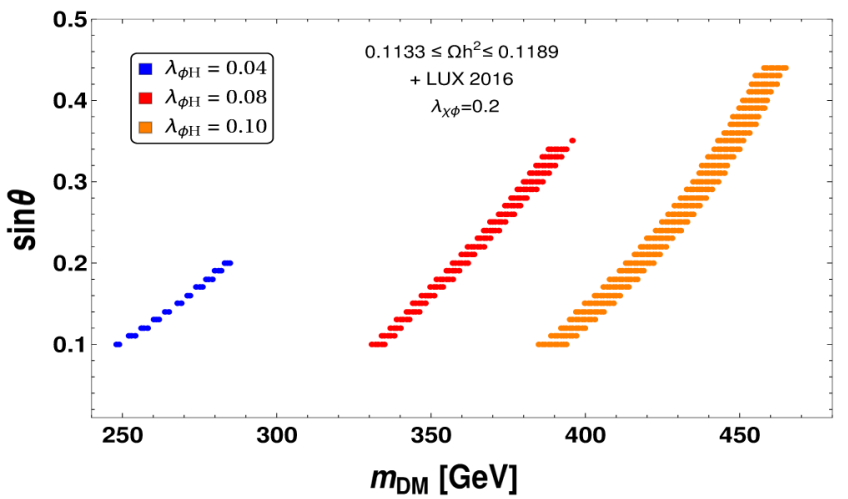

FIG. 10. $m_{\mathrm{DM}}$ vs $\sin \theta$ plot for a fixed $\lambda_{\chi \phi}$ and $\lambda_{\phi H}$ as mentioned in the figure to satisfy the correct relic abundance and direct detection cross section consistent with the LUX 2016 limit. Values of other parameters are $m_{H_{2}}=300 \mathrm{GeV}, \lambda_{\chi \phi}=0.2$, and $v_{\chi}=800 \mathrm{GeV}$.

Finally, we attempt to estimate the $\sin \theta$ required to provide the correct amount of modification over the minimal version of a real singlet DM having interaction with SM Higgs only in order to revive the "below $500 \mathrm{GeV}$ " DM in the picture. In other words, the amount of $\sin \theta$ should be enough to satisfy the correct relic abundance and DD cross section limits of LUX 2016 [24] and XENON 1T [25] for this particular mass range. To do the analysis, we fix $\lambda_{\chi \phi} \sim 0.2$, while three different values of $\lambda_{\phi H}$ at $0.04,0.08$, and 0.10 are considered for the study. We then provide the $\sin \theta$ vs $m_{\text {DM }}$ plot in Fig. 10, which is consistent with relic density and LUX 2016 limits. We infer that a sizable value of $\sin \theta$ is required for this. With $\lambda_{\phi H}=0.1$, we have noted earlier from Fig. 1 that it alone reproduces the desired relic density with a $330 \mathrm{GeV}$ dark matter, although excluded by LUX 2016 limits. Now, we observe from Fig. 10 that in order to make this as a viable DM mass we need to have $\sin \theta=\mathcal{O}(0.1)$ with

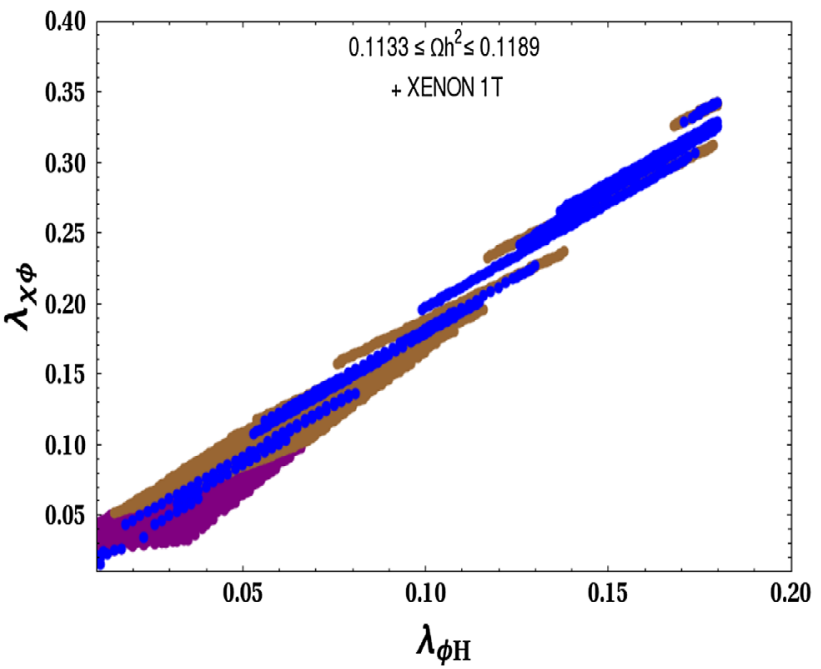

FIG. 9. Relic density satisfied points in the $\lambda_{\phi H^{-}}-\lambda_{\chi \phi}$ plane for $m_{\mathrm{DM}}<150 \mathrm{GeV}$ with the DD cross section consistent with (left panel) LUX 2016 [24] and (right panel) XENON 1T limits [25]. Benchmark points are $m_{\mathrm{H}_{2}}=300 \mathrm{GeV}, v_{\chi}=800 \mathrm{GeV}$, and $\sin \theta=0.2$. 
$\lambda_{\phi H}=0.1$. Such a moderate value of $\sin \theta$ is compatible with LEP and LHC results. A larger value of $\sin \theta \sim \mathcal{O}(0.3)$ with $\lambda_{\phi H}=0.1$ can accommodate DM mass around $440 \mathrm{GeV}$, as seen from Fig. 10. Similarly, we indicate that with $\lambda_{\phi H}=0.08[0.04]$ (for which DM mass $\sim 270 \mathrm{GeV}$ and $110 \mathrm{GeV}$ satisfy the relic density as seen from Fig. 1) $\sin \theta$ variation covers a range of DM mass $\sim 330-370 \mathrm{GeV}$ [240-290 GeV], provided we restrict ourselves to up to $\sin \theta=0.3$.

\section{VACUUM STABILITY}

In this section, we will discuss how the EW vacuum stability can be achieved in our model. For clarification purposes and a comparative study of it, we first discuss how the presence of different ingredients (three RH neutrinos, $\mathrm{DM}$, and the extra scalar $\chi$ ) can affect the running of the Higgs quartic coupling when added one after another. We first comment on the inclusion of the RH neutrinos and investigate the running of $\lambda_{H}$. Then, we study how the involvement of the scalar singlet DM field $\phi$ can alter the conclusion. Finally, we discuss the result corresponding to our setup, i.e., including the $\chi$ field as well.

In doing this analysis, the absolute stability of the Higgs vacuum is ensured by $\lambda_{H}(\mu)>0$ for any energy scale $\mu$ where the EW minimum of the scalar potential is the global minimum. However, there may exist another minimum that is deeper than the EW one. In that case, we need to calculate the tunneling probability of the EW vacuum to the second minimum. The Universe will be in a metastable state, provided the decay time of the EW vacuum is longer than the age of the Universe. The tunneling probability is given by $[5,6]$,

$$
\mathcal{P}=T_{U}^{4} \mu_{B}^{4} e^{-\frac{8 \pi^{2}}{\left.3 \lambda_{H} \mu_{B}\right)},}
$$

where $T_{U}$ is the age of the Universe. $\mu_{B}$ is the scale at which probability is maximized, determined from $\beta_{\lambda_{H}}\left(\mu_{B}\right)=0$. Hence, the metastable Universe requires [5]

$$
\lambda_{H}\left(\mu_{B}\right)>\frac{-0.065}{1-0.01 \ln \left(\frac{v}{\mu_{B}}\right)},
$$

where $T_{U} \simeq 10^{14} \mathrm{yr}$ is used. As noted in Ref. [6], for $\mu_{B}>M_{P}$, one can safely consider $\lambda_{H}\left(\mu_{B}\right)=\lambda_{H}\left(M_{P}\right)$.

Before proceeding further, some discussion on the involvement of light neutrino mass in the context of vacuum stability is pertinent here. As stated before, the light neutrino mass is generated through the type-I seesaw for which three RH neutrinos are included in the setup. We now describe the strategy that we adopt here in order to study their impact on renormalization group (RG) evolution. For simplicity, the RH neutrino mass matrix $M_{N}$ is considered to be diagonal with degenerate entries, i.e., $M_{i=1,2,3}=M_{R}$. As we will see, it is $\operatorname{Tr}\left[Y_{\nu}^{\dagger} Y_{\nu}\right]$ that enters in the $\beta$ function of the relevant couplings. To extract the information on $Y_{\nu}$, we employ the type-I mass formula $m_{\nu}=Y_{\nu}^{T} Y_{\nu} \frac{v^{2}}{2 M_{R}}$. Naively, one would expect that large Yukawas are possible only with very large RH neutrino masses. For example, with $M_{R} \sim 10^{14} \mathrm{GeV}, Y_{\nu}$ comes out to be 0.3 in order to obtain $m_{\nu} \simeq 0.05 \mathrm{eV}$. Contrary to our naive expectation, it can be shown that, even with smaller $M_{R}$, one can achieve large values of $\operatorname{Tr}\left[Y_{\nu}^{\dagger} Y_{\nu}\right]$ once a special flavor structure of $Y_{\nu}$ is considered [37]. Note that we aim to study the EW vacuum stability in the presence of a large value of $\operatorname{Tr}\left[Y_{\nu}^{\dagger} Y_{\nu}\right]$. For this purpose, we use the parametrization by Ref. [90] and write $Y_{\nu}$ as

$$
Y_{\nu}=\sqrt{2} \frac{\sqrt{M_{R}}}{v} \mathcal{R} \sqrt{m_{\nu}^{d}} U_{\mathrm{PMNS}}^{\dagger}
$$

where $m_{\nu}^{d}$ is the diagonal light neutrino mass matrix and $U_{\text {PMNS }}$ is the unitary matrix diagonalizing the neutrino mass matrix $m_{\nu}$ such that $m_{\nu}=U_{\mathrm{PMNS}}^{*} m_{\nu}^{d} U_{\mathrm{PMNS}}^{\dagger}$. Here, $\mathcal{R}$ represents a complex orthogonal matrix that can be written as $\mathcal{R}=O \exp (i \mathcal{A})$ with $O$ as real orthogonal and $\mathcal{A}$ as real antisymmetric matrices, respectively. Hence, one gets

$$
\operatorname{Tr}\left[Y_{\nu}^{\dagger} Y_{\nu}\right]=\frac{2 M_{R}}{v^{2}} \operatorname{Tr}\left[\sqrt{m_{\nu}^{d}} e^{2 i \mathcal{A}} \sqrt{m_{\nu}^{d}}\right] .
$$

Note that the real antisymmetric matrix $\mathcal{A}$ does not appear in the seesaw expression for $m_{\nu}=\frac{Y_{\nu}^{T} Y_{\nu} v^{2}}{2 M_{R}}$. Therefore, with any suitable choice of $\mathcal{A}$, it would actually be possible to have sizeable Yukawas even with light $M_{R}$, and hence this can affect the RG evolution of $\lambda_{H}$ significantly. For an example, let us consider magnitudes of all the entries of $\mathcal{A}$ to be equal, say, $a$ with all diagonal entries as zero. Then, with $M_{R}=1 \mathrm{TeV}, \operatorname{Tr}\left[Y_{\nu}^{\dagger} Y_{\nu}\right]$ can be as large as 1 with $a=8.1[90,91]$. Below, we specify the details of Higgs vacuum stability in the presence of RH neutrinos only.

\section{A. Higgs vacuum stability with right-handed neutrinos}

In the presence of the RH neutrino Yukawa coupling $Y_{\nu}$, the RG equation of SM couplings will be modified [92].

Below, we present the one loop beta functions of Higgs quartic coupling $\lambda_{H}$, top-quark Yukawa coupling $y_{t}$, and neutrino Yukawa coupling $Y_{\nu}$,

$$
\frac{d \lambda_{H}}{d \ln \mu}=\frac{1}{16 \pi^{2}}\left\{\beta_{\lambda_{H}}^{\mathrm{SM}}+\beta_{\lambda_{H}}^{\mathrm{I}}\right\} \quad \text { with } \quad \beta_{\lambda_{H}}^{\mathrm{I}}=4 \lambda_{H} \operatorname{Tr}\left[Y_{\nu}^{\dagger} Y_{\nu}\right]-2 \operatorname{Tr}\left[\left(Y_{\nu}^{\dagger} Y_{\nu}\right)^{2}\right]
$$



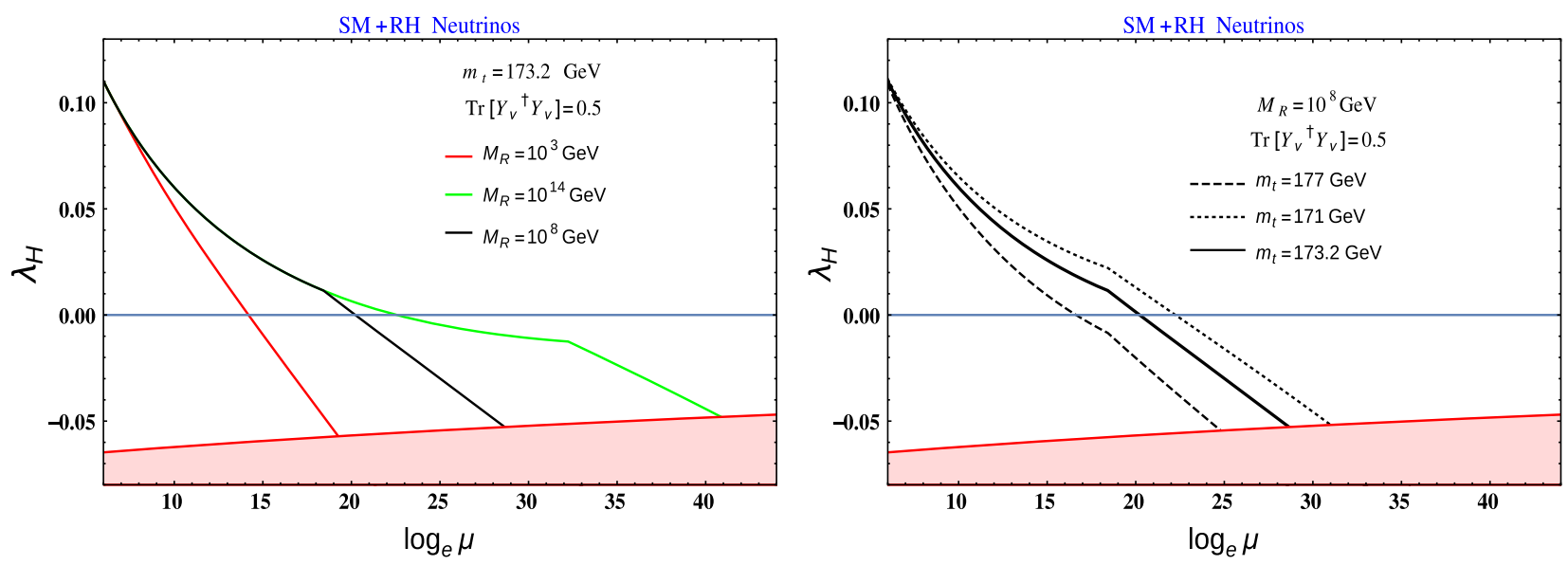

FIG. 11. RG running of $\lambda_{H}$ with energy scale $\mu$ in $\mathrm{SM}+\mathrm{RH}$ neutrinos. (Left panel) Different RH neutrino mass scales $M_{R}$ are considered with fixed $m_{t}=173.2 \mathrm{GeV}$. (Right panel) Different top masses are considered with $M_{R}=10^{8} \mathrm{GeV}$.

$$
\begin{gathered}
\frac{d y_{t}}{d \ln \mu}=\frac{1}{16 \pi^{2}}\left\{\beta_{y_{t}}^{\mathrm{SM}}+\beta_{y_{t}}^{\mathrm{I}}\right\} \quad \text { with } \quad \beta_{y_{t}}^{\mathrm{I}}=\operatorname{Tr}\left[Y_{\nu}^{\dagger} Y_{\nu}\right] y_{t}, \\
\frac{d \operatorname{Tr}\left[Y_{\nu}^{\dagger} Y_{\nu}\right]}{d \ln \mu}=\frac{1}{16 \pi^{2}} \beta_{\operatorname{Tr}\left[Y_{\nu}^{\dagger} Y_{\nu}\right]}^{\mathrm{I}}=\frac{1}{16 \pi^{2}}\left\{\left(6 y_{t}^{2}+2 \operatorname{Tr}\left[Y_{\nu}^{\dagger} Y_{\nu}\right]-\frac{3}{2} g_{1}^{2}-\frac{9}{2} g_{2}^{2}\right) \operatorname{Tr}\left[Y_{\nu}^{\dagger} Y_{\nu}\right]+3 \operatorname{Tr}\left[\left(Y_{\nu}^{\dagger} Y_{\nu}\right)^{2}\right]\right\}
\end{gathered}
$$

where $\beta_{\lambda_{H}}^{\mathrm{SM}}$ and $\beta_{y_{t}}^{\mathrm{SM}}$ represent the $\beta$ functions of $\lambda_{H}$ and $y_{t}$, respectively, in SM. The $Y_{\nu}$ dependence is to be evaluated in accordance with the type-I seesaw expression, $m_{\nu}=Y_{\nu}^{T} Y_{\nu} \frac{v^{2}}{M_{R}}$. Also, with large $a$ (elements of $\mathcal{A}$ ), it is found [37] that $\operatorname{Tr}\left[\left(Y_{\nu}^{\dagger} Y_{\nu}\right)^{2}\right] \simeq \operatorname{Tr}\left[Y_{\nu}^{\dagger} Y_{\nu}\right]^{2}$, and we will be using this approximated relation in obtaining the running of the couplings through Eqs. (21)-(23). Here, we have used the best-fit values of neutrino oscillation parameters for normal hierarchy $[93,94]$. We have also considered the mass of lightest neutrino to be zero.

Note that, just like the top-quark Yukawa coupling, the neutrino Dirac Yukawa has a similar impact on the Higgs quartic coupling, in particular, with large $Y_{\nu}$. Also, the topquark Yukawa would have a contribution dependent on $Y_{\nu}$. This has been studied in several works [36-45]. We summarize here the results with some benchmark values of $\mathrm{RH}$ neutrino masses. These will be useful for a comparative study with the results specific to our model. In Fig. 11 (left panel), we have plotted running of the Higgs quartic coupling $\lambda_{H}$ against energy scale $\mu$ until $M_{P}$ for different choices of $M_{R}=10^{3}, 10^{8}$, and $10^{14} \mathrm{GeV}$ with $\operatorname{Tr}\left[Y_{\nu}^{\dagger} Y_{\nu}\right]=0.5$ denoted by red, black, and green solid lines, respectively. The pink shaded portion represents the instability region given by the inequality $[5] \lambda_{H} \leq-0.065$ / $\left[1-0.01 \ln \left(\frac{v}{\mu}\right)\right]$. As expected, we find that the Higgs quartic coupling enters into the instability region well before the Planck scale.

In the right panel of Fig. 11, the effect of choosing different $m_{t}$ within the present $2 \sigma$ uncertainty is shown for a fixed $M_{R}=10^{8} \mathrm{GeV}$. The black solid, dashed, and dotted lines represent the $\lambda_{H}$ running with $m_{t}$ as 173.2, 177, and $171 \mathrm{GeV}$, respectively. In doing this analysis, we fix the initial values of all SM couplings [6] as given in Table I at an energy scale $\mu=m_{t}$. Here, we consider $m_{h}=125.09 \mathrm{GeV}, m_{t}=173.2 \mathrm{GeV}$, and $\alpha_{s}=0.1184$. In Fig. 12, we have shown a region plot for $\operatorname{Tr}\left[Y_{\nu}^{\dagger} Y_{\nu}\right]$ and $m_{t}$ with fixed $M_{R}$ at $10^{8} \mathrm{GeV}$ in terms of stability $\left(\lambda_{H}\right.$ remains positive all the way up to $M_{P}$ ), metastability, and instability of the EW vacuum of the SM. The top-quark mass is varied between 168 and $178 \mathrm{GeV}$. The region in which the EW vacuum is stable is indicated by green, and the metastable region is indicated by white patches. The instability region is denoted by the pink shaded part. It can be noted that the result coincides with the one obtained in Ref. [40]. We aim to discuss the change obtained over this diagram in the context of our model.

\section{B. Higgs vacuum stability from Higgs portal DM and RH neutrinos}

Here, we discuss the vacuum stability scenario in the presence of both the scalar DM $(\phi)$ and three $\mathrm{RH}$

TABLE I. Values of the relevant SM couplings (top-quark Yukawa $y_{t}$, gauge couplings $g_{i}(i=1,2,3)$ and Higgs quartic coupling $\lambda_{H}$ ) at energy scale $\mu=m_{t}=173.2 \mathrm{GeV}$ with $m_{h}=$ $125.09 \mathrm{GeV}$ and $\alpha_{S}\left(m_{Z}\right)=0.1184$.

\begin{tabular}{lccccc}
\hline \hline Scale & $y_{t}$ & $g_{1}$ & $g_{2}$ & $g_{3}$ & $\lambda_{H}$ \\
\hline$\mu=m_{t}$ & 0.93610 & 0.357606 & 0.648216 & 1.16655 & 0.125932 \\
\hline \hline
\end{tabular}




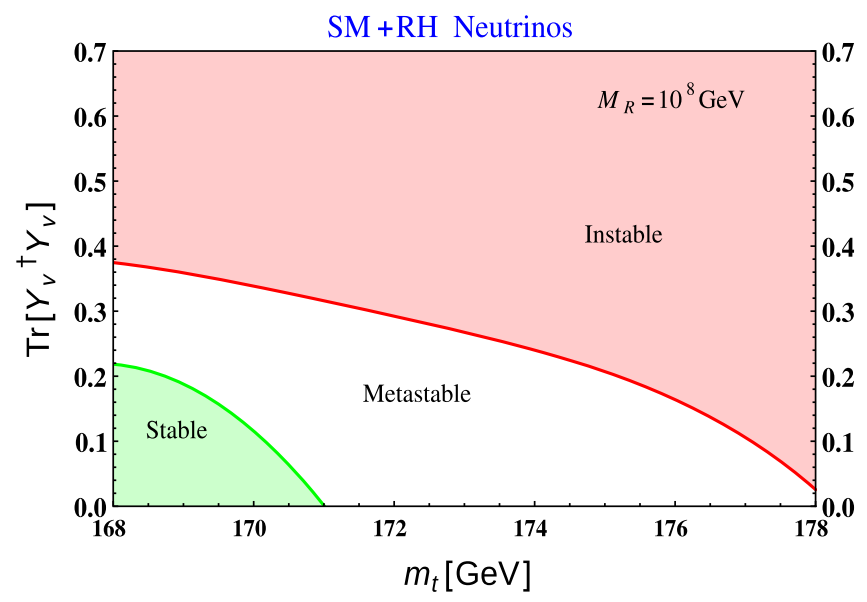

FIG. 12. Region plot for $m_{t}-\operatorname{Tr}\left[Y_{\nu}^{\dagger} Y_{\nu}\right]$ in the SM, extended with $\mathrm{RH}$ neutrinos having degenerate mass $M_{R}=10^{8} \mathrm{GeV}$. The plane is divided into three categories: i) absolute stability, ii) metastability, and iii) instability.

neutrinos $(N)$. In that case, the effective scalar potential becomes $V_{\mathrm{I}}+V_{H}$ only. Note that the DM phenomenology is essentially unaffected from the inclusion of the heavy $\mathrm{RH}$ neutrinos with the assumption $M_{R} \gg m_{\mathrm{DM}}$. On the other hand, combining Eqs. (21)-(23), we obtain the corresponding beta functions for the couplings as provided below;

$$
\begin{aligned}
\frac{d \lambda_{H}}{d t}= & \frac{1}{16 \pi^{2}}\left\{\beta_{\lambda_{H}}^{\mathrm{SM}}+\beta_{\lambda_{H}}^{\mathrm{I}}+\beta_{\lambda_{H}}^{\mathrm{II}}\right\} \quad \text { where } \beta_{\lambda_{H}}^{\mathrm{II}}=\frac{\lambda_{\phi H}^{2}}{2}, \\
\frac{d \lambda_{\phi H}}{d t}= & \frac{1}{16 \pi^{2}} \beta_{\lambda_{\phi H}}^{\mathrm{I}} \\
= & \frac{1}{16 \pi^{2}}\left\{12 \lambda_{H} \lambda_{\phi H}+\lambda_{\phi} \lambda_{\phi H}+4 \lambda_{\phi H}^{2}+6 y_{t}^{2} \lambda_{\phi H}\right. \\
& \left.-\frac{3}{2} g_{1}^{2} \lambda_{\phi H}-\frac{9}{2} g_{2}^{2} \lambda_{\chi H}+2 \operatorname{Tr}\left[Y_{\nu}^{\dagger} Y_{\nu}\right] \lambda_{\phi H}\right\}, \\
& \frac{d \lambda_{\phi}}{d t}=\frac{1}{16 \pi^{2}} \beta_{\lambda_{\phi}}^{\mathrm{I}}=\frac{1}{16 \pi^{2}}\left\{3 \lambda_{\phi}^{2}+12 \lambda_{\phi H}^{2}\right\} .
\end{aligned}
$$

From the additional term $\beta_{\lambda_{H}}^{\mathrm{II}}$, we expect that the involvement of DM would affect the EW vacuum stability in a positive way (i.e., pushing the vacuum more toward the stability) as shown in Refs. [29-34], whereas we noted in the previous subsection that the Yukawa coupling (if sizable) has a negative impact on it.

The interplay between the neutrino Yukawa coupling and Higgs portal coupling with DM is shown in Fig. 13, left and right panels (top and bottom). For the purpose of comparison, we have kept the same set of choices of parameters as in Fig. 11 (left and right panels). For the top panels, we consider mass of the dark matter to be $m_{\mathrm{DM}}=300 \mathrm{GeV}$, and for the bottom set, $m_{\mathrm{DM}}=920 \mathrm{GeV}$ is taken. The choice of $m_{\mathrm{DM}}$ could in turn fix the $\lambda_{\phi H}$ coupling from the relic density plot of Fig. 1. For example, with $m_{\mathrm{DM}}=300 \mathrm{GeV}, \lambda_{\phi H}$ is 0.075 , and for $m_{\mathrm{DM}}=$ $920 \mathrm{GeV}, \lambda_{\phi H}$ is given by a 0.286 value. It is evident that the presence of Higgs portal coupling only has a mild effect as compared to the impact created by the neutrino Yukawa coupling. Finally, in Fig. 14, we provide the region plot in the $\operatorname{Tr}\left[Y_{\nu}^{\dagger} Y_{\nu}\right]-m_{t}$ plane in which the stable and instable regions are indicated by green and pink patches. This plot, while compared with Fig. 12, indicates that there is no such noticeable improvement except the mild enhancement of the metastable region due to the involvement of singlet scalar (DM) with Higgs portal coupling. With an aim to accommodate both the massive neutrinos and a relatively light dark matter $(<500 \mathrm{GeV})$, we move on to the next section in which the $\chi$ field is included.

\section{EW vacuum stability in extended Higgs portal DM and RH neutrinos}

Turning into the discussion on vacuum stability in our framework of the extended Higgs portal having three RH neutrinos, DM, and the $\chi$ fields, we first put together the relevant RG equations (for $\mu>m_{\mathrm{DM}}, m_{\mathrm{H}_{2}}$ ) as given by

$$
\begin{gathered}
\frac{d \lambda_{H}}{d t}=\frac{1}{16 \pi^{2}}\left\{\beta_{\lambda_{H}}^{\mathrm{SM}}+\beta_{\lambda_{H}}^{\mathrm{I}}+\beta_{\lambda_{H}}^{\mathrm{II}}+\frac{\lambda_{\chi H}^{2}}{2}\right\} \\
\frac{d \lambda_{\phi H}}{d t}=\frac{1}{16 \pi^{2}}\left\{\beta_{\lambda_{\phi H}}^{\mathrm{I}}+\lambda_{\chi \phi} \lambda_{\chi H}\right\} \\
\frac{d \lambda_{\phi}}{d t}=\frac{1}{16 \pi^{2}}\left\{\beta_{\lambda_{\phi}}^{\mathrm{I}}+3 \lambda_{\chi \phi}^{2}\right\} \\
\frac{d \lambda_{\chi H}}{d t}=\frac{1}{16 \pi^{2}}\left\{12 \lambda_{H} \lambda_{\chi H}+\lambda_{\chi} \lambda_{\chi H}+4 \lambda_{\chi H}^{2}+6 y_{t}^{2} \lambda_{\chi H}\right. \\
\left.-\frac{3}{2} g_{1}^{2} \lambda_{\chi H}-\frac{9}{2} g_{2}^{2} \lambda_{\chi H}+\lambda_{\chi \phi} \lambda_{\phi H}+2 \operatorname{Tr}\left[Y_{\nu}^{\dagger} Y_{\nu}\right] \lambda_{\chi H}\right\} \\
\frac{d \lambda_{\chi}}{d t}=\frac{1}{16 \pi^{2}}\left\{3 \lambda_{\chi}^{2}+12 \lambda_{\chi H}^{2}+3 \lambda_{\chi \phi}^{2}\right\} \\
\frac{d \lambda_{\chi \phi}}{d t}=\frac{1}{16 \pi^{2}}\left\{4 \lambda_{\chi \phi}^{2}+\lambda_{\chi \phi}\left(\lambda_{\phi}+\lambda_{\chi}\right)+4 \lambda_{\phi H} \lambda_{\chi H}\right\}
\end{gathered}
$$

We note that the couplings $\lambda_{\chi \phi}, \lambda_{\phi H}$, and $\lambda_{\chi H}$, which played an important role in DM phenomenology, are involved in the running of couplings as well. From the discussion of the DM section, we have estimated these parameters in a range so as to satisfy the appropriate relic density and be within the direct search limits for a specific choice of other parameters at their reference values: $m_{H_{2}}=300 \mathrm{GeV}$ and $v_{\chi}=800 \mathrm{GeV}$ and $\sin \theta=0.2$ (henceforth, we describe this set as A). In particular, an estimates for $\lambda_{\chi \phi}, \lambda_{\phi H}$ are obtained from Fig. 4 (for $150 \mathrm{GeV}<m_{\mathrm{DM}}<500 \mathrm{GeV}$ ) and from Fig. 9 (for $m_{\mathrm{DM}}<150 \mathrm{GeV}$ ), having different choices of $m_{\mathrm{DM}}$ and 

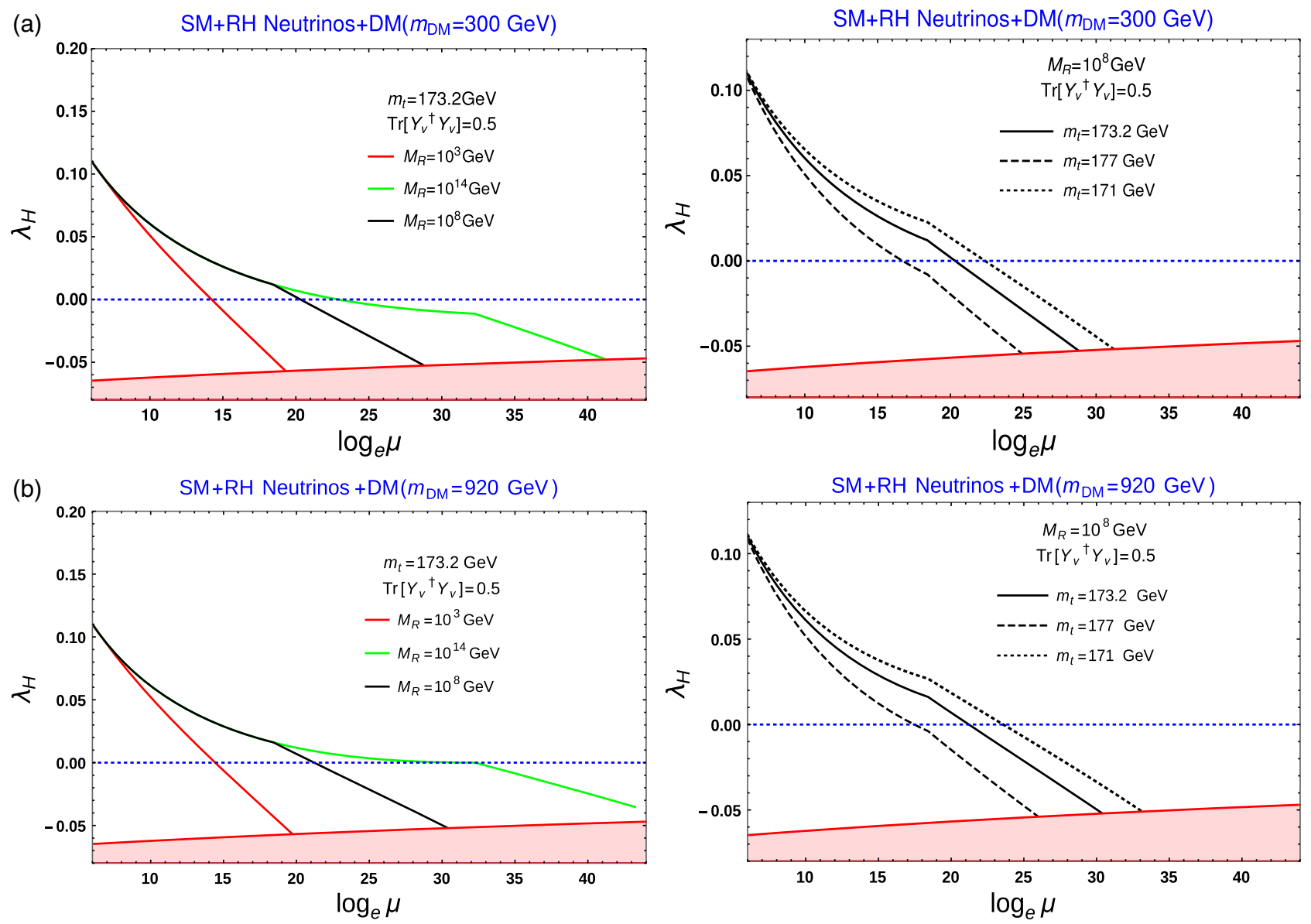

FIG. 13. RG evolution of $\lambda_{H}$ with energy scale $\mu$ with $\mathrm{SM}+\mathrm{DM}+\mathrm{RH}$ neutrinos with $\lambda_{\phi}=0.7, m_{H}=125.09 \mathrm{GeV}$, and $\alpha_{S}\left(m_{Z}\right)=0.1184$.: (a) (top panels) $m_{\mathrm{DM}}=300 \mathrm{GeV}$ and (b) (bottom panels) $m_{\mathrm{DM}}=920 \mathrm{GeV}$. In the left panels, $m_{t}$ is fixed at $173.2 \mathrm{GeV}$, and plots are there with different $M_{R}$, while in right panel, $M_{R}$ is fixed at $10^{8} \mathrm{GeV}$, and different $m_{t}$ values are considered.
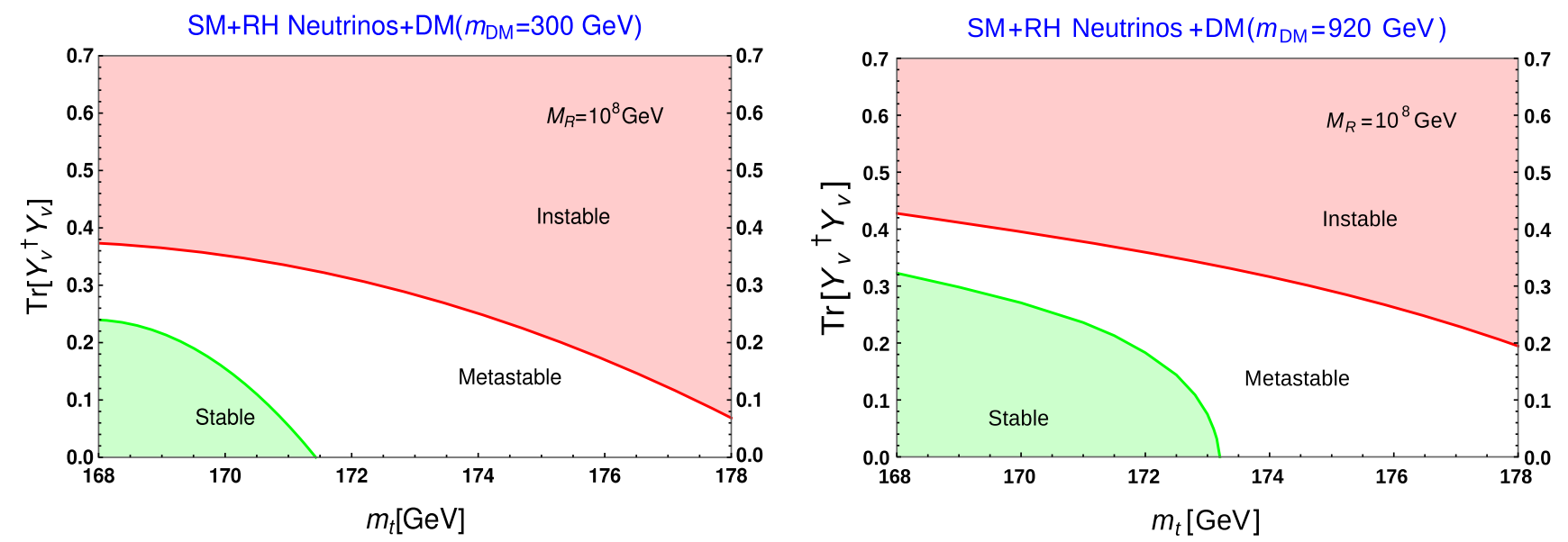

FIG. 14. Regions of stability, metastability, and instability in the $\mathrm{SM}+\mathrm{DM}+\mathrm{RH}$ neutrinos case in the $\operatorname{Tr}\left[Y_{\nu}^{\dagger} Y_{\nu}\right]-m_{t}$ plane for $m_{\mathrm{DM}}=$ $300 \mathrm{GeV}$ (left panel) and $920 \mathrm{GeV}$ (right panel). We consider $\lambda_{\phi}=0.7, m_{H}=125.09 \mathrm{GeV}$, and $\alpha_{S}\left(m_{Z}\right)=0.1184$ for both the figures.

$\sin \theta$. The parameter $\lambda_{\chi H}$ dependence is mostly realized through $\sin \theta$ following Eq. (10), in which $m_{\mathrm{H}_{2}}$ and $\tan \beta$ are fixed from set $\mathrm{A}$. This $\sin \theta$ is the most crucial parameter that controls both the DM phenomenology and the vacuum stability. We have already seen that it allows the scalar singlet DM to be viable for the low-mass window by relaxing $\lambda_{\phi H}$ from its sole role in the case of single scalar singlet DM. On the other hand, a nonzero $\sin \theta$ provides a 

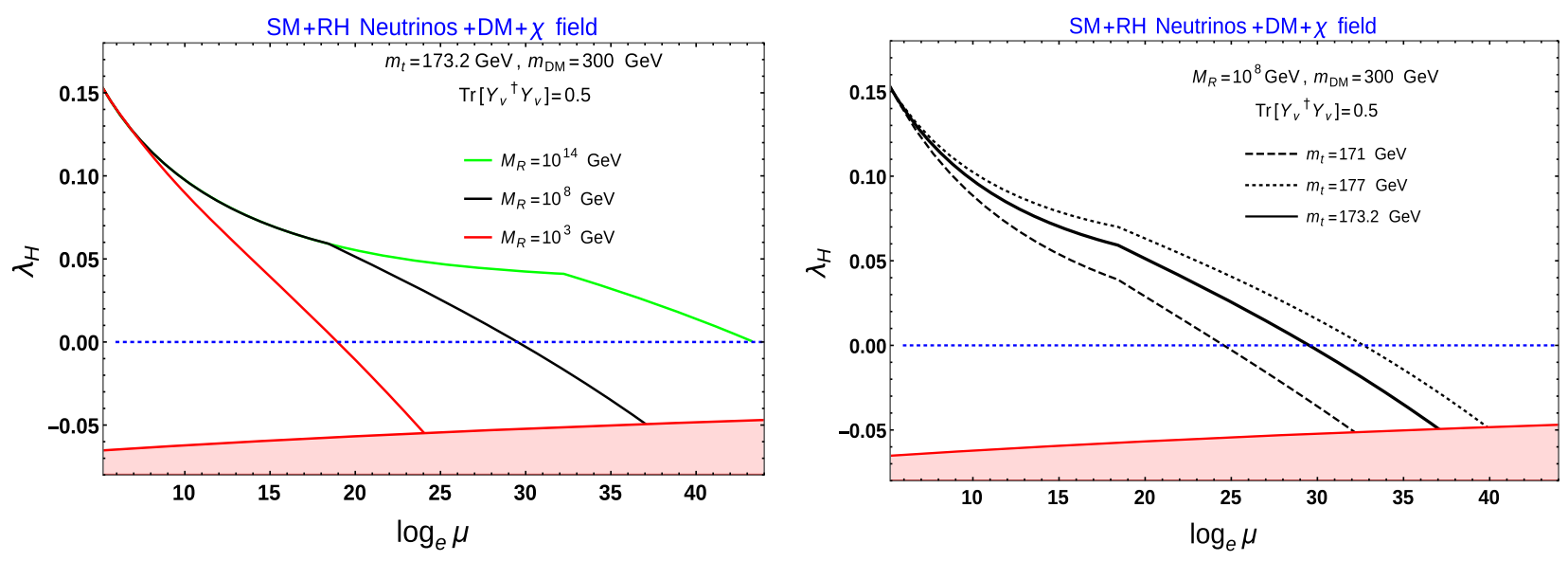

FIG. 15. RG running of $\lambda_{H}$ vs $\mu$ in the combined scenario of $\mathrm{SM}+\mathrm{RH}$ neutrinos $+\mathrm{DM}+\chi$ field with $m_{\mathrm{DM}}=300 \mathrm{GeV}$, $\sin \theta=0.2$, and $m_{H_{2}}=300 \mathrm{GeV}$. In the left panel, $m_{t}(\sim 173.2 \mathrm{GeV})$ is kept fixed, and $M_{R}$ is varied, and in the right panel, $M_{R}\left(\sim 10^{8} \mathrm{GeV}\right)$ is fixed, and $m_{t}$ has been varied. Point $\mathrm{X}\left(\lambda_{\phi H}=0.06, \lambda_{\chi \phi}=0.135\right)$ from Fig. 8 and $\lambda_{\phi}=0.7$ have been used as benchmark points.

positive shift [it is effectively the threshold effect in the small- $\theta$ limit as seen from Eq. (8)] to the Higgs quartic coupling and hence guides the $\lambda_{H}$ toward more stability. Hence, $\sin \theta$ would be a crucial parameter in this study. Note that the RH neutrinos being relatively heavy as compared to the DM, neutrino Yukawa coupling does not play much role in DM phenomenology.

Assuming the validity of this extended SM (with three RH neutrinos and two singlets, $\phi$ and $c h i$ ) up to the Planck scale, we study the running of the Higgs quartic coupling $\lambda_{H}$ from the EW scale to $M_{P}$ as shown in Fig. 15. In obtaining the running, we have considered $m_{\mathrm{H}_{2}}=300 \mathrm{GeV}$, $\sin \theta=0.2$, and $m_{\mathrm{DM}}$ is considered to be $300 \mathrm{GeV}$. The values of $\lambda_{\chi \phi}$ and $\lambda_{\phi H}$ are fixed at 0.135 and 0.06 , respectively (this particular point is denoted by a blue dot, named $x$, in Fig. 8). It turns out that any other set of $\lambda_{\chi \phi}$ and $\lambda_{\phi H}$ other than this blue dot from Fig. 8 (while
$m_{\mathrm{DM}}=300 \mathrm{GeV}$ is fixed) would not change our conclusion significantly as long as $\sin \theta$ is considered at 0.2 . To compare the effect of the extra scalar $\chi$ in the theory, we keep the neutrino parameters $\operatorname{Tr}\left[Y_{\nu}^{\dagger} Y_{\nu}\right]$ and $M_{R}$ at their respective values considered in Figs. 11 and 13.

In the left panel of Fig. 15, the running is performed for three different choices of $M_{R}$, specifically at $1 \mathrm{TeV}$, $10^{8} \mathrm{GeV}$, and $10^{14} \mathrm{GeV}$, while the top mass is fixed at 173.2 GeV. A similar plot is exercised in right panel of Fig. 15, in which three different choices of $m_{t}=$ $(171,173.2,177) \mathrm{GeV}$ are considered, while $M_{R}$ is fixed at $10^{8} \mathrm{GeV}$. Contrary to our previous finding in Sec. V.A and V.B (see Figs. 11 and 13), we clearly see here that with $M_{R}=10^{14} \mathrm{GeV}$ and $m_{t}=171 \mathrm{GeV} \lambda_{H}$ remains positive up to $M_{P}$ even in the presence of large $\operatorname{Tr}\left[Y_{\nu}^{\dagger} Y_{\nu}\right] \sim \mathcal{O}(1)$. Hence, the EW vacuum turns out to be absolutely stable. Although there exist other values of $M_{R}$ and/or $m_{t}$, for

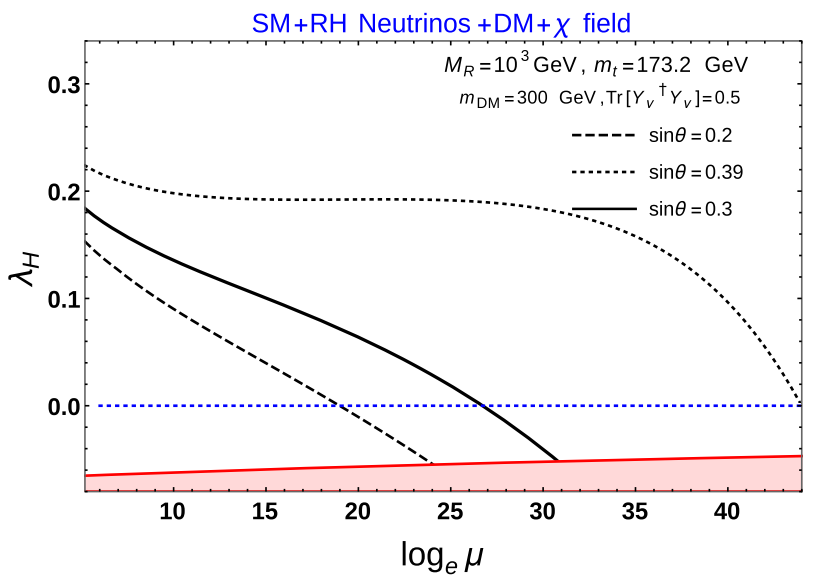

FIG. 16. RG running of $\lambda_{H}$ with energy scale $\mu$ for different values of $\sin \theta$ in the combined setup of SM $+\mathrm{DM}+\mathrm{RH}$ neutrinos $+\chi$ field where in the left panel $M_{R}=10^{8} \mathrm{GeV}$ and in the right panel $M_{R}=10^{3} \mathrm{GeV}$. Other reference values are $m_{\mathrm{DM}}=300 \mathrm{GeV}$, $m_{H_{2}}=300 \mathrm{GeV}, \operatorname{Tr}\left[Y_{\nu}^{\dagger} Y_{\nu}\right]=0.5, \lambda_{\phi H}=0.06$, and $\lambda_{\chi \phi}=0.135$. 


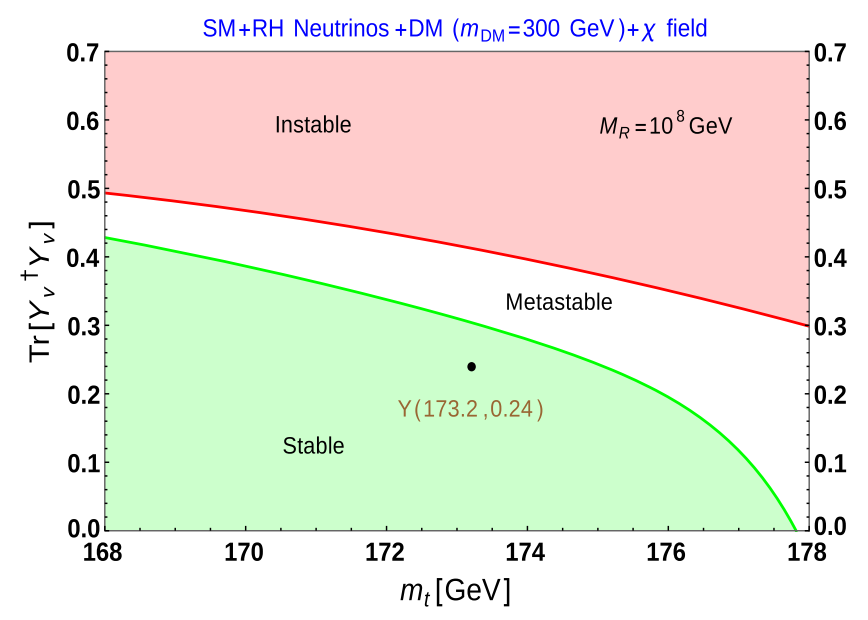

FIG. 17. Stability, metastability, and instability region on the $\operatorname{Tr}\left[Y_{\nu}^{\dagger} Y_{\nu}\right]-m_{t}$ plane for $M_{R}=10^{8} \mathrm{GeV}$ in the extended scenario of SM with three $\mathrm{RH}$ neutrinos, DM, and $\chi$. We have used point $\mathrm{X}\left(\lambda_{\phi H}=0.06, \lambda_{\chi \phi}=0.135\right)$ from Fig. $8, \sin \theta=0.2$, $m_{\mathrm{H}_{2}}=300 \mathrm{GeV}, v_{\chi}=800 \mathrm{GeV}, m_{\mathrm{DM}}=300 \mathrm{GeV}$, and $\lambda_{\phi}=0.7$ as benchmark points.

which the EW vacuum still remains unstable, the scale at which $\lambda_{H}$ enters into the instable region is getting delayed with a noticeable change from earlier cases (Figs. 11 and 13). This becomes possible due to the introduction of the $\chi$ field having contribution mostly from the $\sin \theta$ parameter. To show its impact on stability, in Fig. 16 (left panel), we plot $\lambda_{H}$ running with different choices of $\sin \theta=0.1,0.2$, 0.3 for $M_{R}=10^{8} \mathrm{GeV}, m_{t}=173.2 \mathrm{GeV}$, and $m_{\mathrm{DM}}=$ $300 \mathrm{GeV}$ while keeping $\operatorname{Tr}\left[Y_{\nu} Y_{\nu}^{\dagger}\right]=0.5$ (same as in Fig. 15, left panel, black solid line). It shows that, while $\sin \theta=0.2$ (black solid line) cannot make the EW vacuum absolutely stable until $M_{P}$, an increase of the $\sin \theta$ value $\sim 0.3$ can do it (dotted line). Similarly, in Fig. 16 (right panel), we consider a lower $M_{R}$ as $1 \mathrm{TeV}$. We have already noticed that such a low $M_{R}$ with large $\operatorname{Tr}\left[Y_{\nu}^{\dagger} Y_{\nu}\right]=0.5$ pushes the EW vacuum toward instability at a much lower scale $\sim 10^{6} \mathrm{GeV}$. To make the EW vacuum stable with such an $M_{R}$ and $\operatorname{Tr}\left[Y_{\nu}^{\dagger} Y_{\nu}\right]$, one requires $\sin \theta \sim 0.4$ as seen from the right panel of Fig. 16 (dotted line). However, such a large $\sin \theta$ is ruled out from the experimental constraints [77]. For representative purposes, we also include the study with other $\sin \theta=0.2,0.3$ denoted by dashed and solid lines.

We provide Fig. 17, in which the regions with stability, metastability, and instability are marked as green, white, and pink patches in the plane containing $\operatorname{Tr}\left[Y_{\nu}^{\dagger} Y_{\nu}\right]$ and $m_{t}$. With $M_{R}=10^{3} \mathrm{GeV}$ and $M_{R}=10^{14} \mathrm{GeV}$, similar plots are shown in Fig. 18, left and right panels. Finally, in Fig. 19, we have shown the RG evolution of all the stability conditions in Eq. (11) from $m_{t}$ to $M_{P}$ to check their validity all the way up to $M_{P}$. For this purpose, we have considered the initial values of the parameters involved in the following way. For values of $\lambda_{\phi H}$ and $\lambda_{\chi \phi}$ corresponding to $\sin \theta=0.2, v_{\chi}=800 \mathrm{GeV}$, and $m_{\mathrm{DM}}=300 \mathrm{GeV}$, we have considered the benchmark point values as indicated by a blue dot named $X$ in Fig. 8. The value of $\lambda_{\chi}$ is then followed from Eq. (9), and $\lambda_{\phi}$ is chosen to be at 0.7. Values of $\operatorname{Tr}\left[Y_{\nu}^{\dagger} Y_{\nu}\right]=0.24$ and $m_{t}=173.2 \mathrm{GeV}$ are chosen for this purpose from Fig. 17 (here, the benchmark values are denoted by a black dot Y). We conclude that all the stability criteria are fulfilled within the framework. Lastly, we comment that, instead of picking up the point $\mathrm{X}$ from the relic density contour with $\sin \theta \sim 0.2$ in Fig. 8 to study vacuum stability in our model, we could have chosen any other point from that curve. As the stability of the Higgs vacuum primarily depends on the value of $\theta$, our conclusion would not change much. However, the choice of any point having large $\lambda_{\chi \phi}$ could make it, reaching Landau the pole well before $M_{P}$ in its RG running through Eq. (30). To avoid that, one can reduce the value of $\lambda_{\phi} \sim \mathcal{O}\left(10^{-2}\right)$ or less
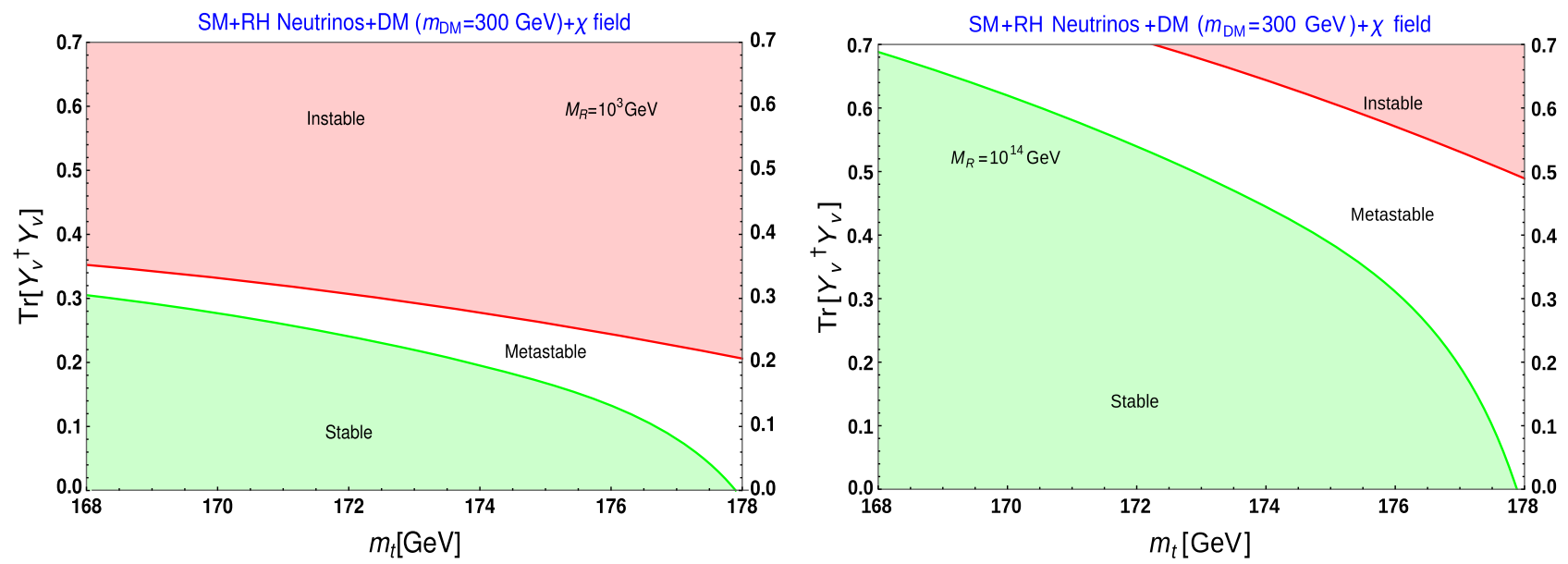

FIG. 18. Stability, metastability, and instability regions on $\operatorname{Tr}\left[Y_{\nu}^{\dagger} Y_{\nu}\right]-m_{t}$ plane in the extended scenario of SM with 3 RH neutrinos, DM and $\chi$ for (left panel) $M_{R}=10^{3} \mathrm{GeV}$ and (right panel) $M_{R}=10^{14} \mathrm{GeV}$. We have used the reference point X $\left(\lambda_{\phi H}=0.06, \lambda_{\chi \phi}=0.135\right)$ from Fig. 8 along with $\sin \theta=0.2, m_{H_{2}}=300 \mathrm{GeV}, v_{\chi}=800 \mathrm{GeV}, m_{\mathrm{DM}}=300 \mathrm{GeV}$ and $\lambda_{\phi}=0.7$ as benchmark values. 


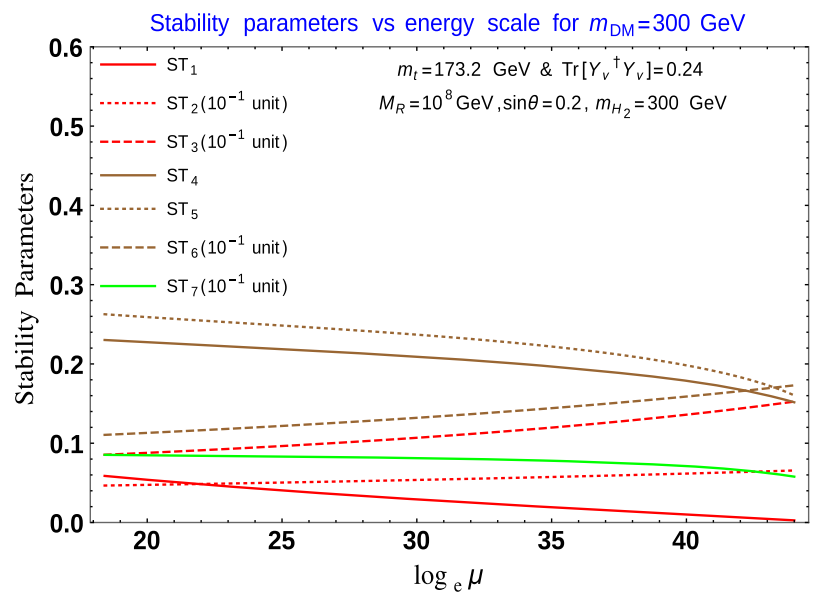

FIG. 19. Evolution of stability parameters [Eq. (11)] for the point $\mathrm{Y}\left(m_{t}=173.2 \mathrm{GeV}, \operatorname{Tr}\left[Y_{\nu}^{\dagger} Y_{\nu}\right]=0.24\right)$ from Fig. 18 (top-right panel). Benchmark points: Point $\mathrm{X}\left(\lambda_{\phi H}=0.06\right.$ and $\left.\lambda_{\chi \phi}=0.135\right)$ from Fig. 8, $M_{R}=10^{8} \mathrm{GeV}, \sin \theta=0.2, m_{H_{2}}=300 \mathrm{GeV}$, $v_{\chi}=800 \mathrm{GeV}, m_{\mathrm{DM}}=300 \mathrm{GeV}$, and $\lambda_{\phi}=0.7$ have been used.

(earlier, it was 0.7), which has no direct connection to or impact on DM phenomenology and vacuum stability analysis in the proposed setup. In Fig. 20, we have shown the running of all parameters from $M_{R}$ to $M_{P}$ involved in perturbative unitarity bound for the benchmark point: $m_{H_{2}}=300 \mathrm{GeV}, \tan \beta=0.30, \sin \theta=0.2, m_{\mathrm{DM}}=300 \mathrm{GeV}$, $\lambda_{\phi H}=0.06, \lambda_{\chi \phi}=0.135, M_{R}=10^{8} \mathrm{GeV}$, and $\operatorname{Tr}\left[Y_{\nu}^{\dagger} Y_{\nu}\right]=$ 0.24 with $m_{t}=173.2 \mathrm{GeV}$. The parameters never exceed the upper limits coming from the unitarity bound. We have also confirmed that any other benchmark points wherever mentioned in our analysis satisfy the perturbativity unitarity limit.

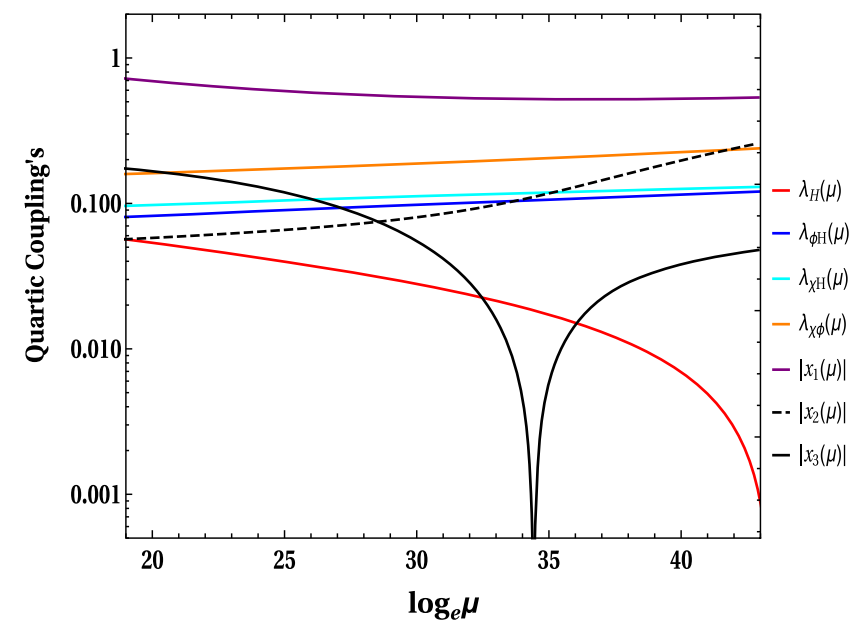

FIG. 20. Evolution of parameters required to satisfy the perturbativity unitarity limit [Eq. (12)] for the point $\mathrm{Y}\left(m_{t}=173.2 \mathrm{GeV}\right.$, $\left.\operatorname{Tr}\left[Y_{\nu}^{\dagger} Y_{\nu}\right]=0.24\right)$ from Fig. 18 (top-right panel). Benchmark points: Point $\mathrm{X}\left(\lambda_{\phi H}=0.06, \lambda_{\chi \phi}=0.135\right)$ from Fig. 8, $M_{R}=10^{8} \mathrm{GeV}, \sin \theta=0.2, m_{H_{2}}=300 \mathrm{GeV}, v_{\chi}=800 \mathrm{GeV}$, $m_{\mathrm{DM}}=300 \mathrm{GeV}$, and $\lambda_{\phi}=0.7$ have been considered.
We end this section by comparing the results of vacuum stability in presence of i) only $\mathrm{RH}$ neutrinos, ii) $\mathrm{RH}$ neutrinos + DM, and iii) $\mathrm{RH}$ neutrinos $+\mathrm{DM}+$ extra scalar with nonzero vev, where in each case neutrino Yukawa coupling $Y_{\nu}$ has sizeable contributions. For this purpose, we consider $m_{t}=173.2 \mathrm{GeV}$ and $M_{R}=10^{8} \mathrm{GeV}$. From Fig. 12, for SM + RH neutrinos, we see that stability cannot be achieved. The metastability scenario is still valid in this case up to $\operatorname{Tr}\left[Y_{\nu}^{\dagger} Y_{\nu}\right]<0.26$. Next, we add a singlet scalar DM candidate with nonzero Higgs portal coupling to SM with RH neutrinos. Figure 14 (left panel) shows, for $m_{\mathrm{DM}}=300 \mathrm{GeV}$, stability of the EW vacuum still remains elusive. On the other hand, the metastability bound on $\operatorname{Tr}\left[Y_{\nu}^{\dagger} Y_{\nu}\right]$ increases slightly from the previous limit to 0.28 . So, DM with mass $300 \mathrm{GeV}$ has a mild impact on study of vacuum stability. Finally, we add the extra scalar singlet with a nonzero vev to the SM with RH neutrinos and scalar DM. We have fixed the heavier Higgs mass $m_{\mathrm{H}_{2}}=$ $300 \mathrm{GeV}$ and $\sin \theta=0.2$. Now, in the combined setup of the $\mathrm{SM}$, scalar DM, scalar with a nonzero vev, and RH neutrinos, the situation changes drastically from the previous case, as seen in Fig. 17. For the same top and RH neutrino masses, we can now achieve absolute stability up to $\operatorname{Tr}\left[Y_{\nu}^{\dagger} Y_{\nu}\right]<0.3$ and the metastability bound on $\operatorname{Tr}\left[Y_{\nu}^{\dagger} Y_{\nu}\right]$ further improved to 0.41 . Overall notable enhancement in the stability and metastability region has been observed in the $\operatorname{Tr}\left[Y_{\nu}^{\dagger} Y_{\nu}\right]-m_{t}$ plane compared to the earlier cases. Hence, the numerical comparison clearly shows that the extra scalar having nonzero mixing with SM Higgs effectively plays the leading role to get absolute vacuum stability in our model.

\section{CONNECTION WITH OTHER OBSERVABLES}

In this section, we first discuss in brief the constraints on the parameters of the model that may arise from lepton flavor-violating (LFV) decays. The most stringent limit follows from the $\mu \rightarrow e \gamma$ decay process. The branching ratio of such a decay process in our setup is given by [95-97]

$$
\operatorname{Br}(\mu \rightarrow e \gamma)=\frac{3 \alpha_{e} v^{4}}{16 \pi M_{R}^{4}}\left|Y_{\nu_{e i}}^{\dagger} Y_{\nu_{i \mu}}\right|^{2}|f(x)|^{2},
$$

where $\alpha_{e}=\frac{e^{2}}{4 \pi}$ is the fine structure constant, $i$ runs from 1 to $3, x=\frac{M_{R}^{2}}{m_{W}^{2}}$, and

$$
f(x)=\frac{x\left(2 x^{3}+3 x^{2}-6 x-6 x^{2} \ln x+1\right)}{2(1-x)^{4}} .
$$

The current experimental limit on the LFV branching ratio is [4]

$$
\operatorname{Br}(\mu \rightarrow e \gamma)<5.7 \times 10^{-13} .
$$

Using this limit, we therefore obtain bounds on $\left|\left(Y_{\nu}^{\dagger} Y_{\nu}\right)_{e \mu}\right|$ corresponding to a fixed $M_{R}$ value that can be converted to constrain $\operatorname{Tr}\left[Y_{\nu}^{\dagger} Y_{\nu}\right]$ in our setup. In obtaining limits on $\operatorname{Tr}\left[Y_{\nu}^{\dagger} Y_{\nu}\right]$ (for fixed $M_{R}$ ), first note that 
$Y_{\nu}^{\dagger} Y_{\nu}$ remains a function of $M_{R}$ and parameter $a$ only [see Eq. (19) with $O=$ !], once the best-fit values of neutrino mixing angles $[93,94]$ are used to evaluate $U_{\text {PMNS }}$. Hence, the LFV limit basically constrains the parameter $a$, which in turn is used to obtain $\operatorname{Tr}\left[Y_{\nu}^{\dagger} Y_{\nu}\right]$. This limit is shown in Fig. 21 by the brown solid line, the left side of which is the region disallowed by lepton-flavor violation.

In the same plane of Fig. 21, we also include the region of the parameter space allowed by both stability and metastability criteria. The green shaded region denotes the absolute stability of the Higgs vacuum, while the white region satisfies the metastability condition. We also indicate the instability region by the pink patch in the same figure under discussion. For this purpose, we have used $m_{t}=173.2 \mathrm{GeV}, m_{\mathrm{DM}}=300 \mathrm{GeV}, \sin \theta=0.2, \lambda_{\phi H}=0.06$, and $\lambda_{\chi \phi}=0.135$ (corresponding to the benchmark point indicated by $\mathrm{X}$ in Fig. 8). The brown shaded region is disfavored by the LFV constraint. Hence, from Fig. 21, we infer that for low- $M_{R}$ LFV constraints turn out to be stronger and for high $M_{R}$ values $\operatorname{Tr}\left[Y_{\nu}^{\dagger} Y_{\nu}\right]$ is mostly restricted by the stability issue.

It turns out that the proposed scenario does not provide any significant contribution to neutrinoless double beta decay [98-103] even for relatively low RH neutrino mass $\left(\sim 10^{3} \mathrm{GeV}\right)$. This is in line with the observation made in Ref. [39]. Before concluding the section, it is perhaps important to comment on the possibility of explaining the baryon asymmetry of the Universe (BAU). The involvement of RH neutrinos would make the leptogenesis a natural candidate to explain BAU from the completion point of view. However, with the exactly degenerate RH neutrinos (we consider this for simplicity though), it is not possible. Once a small-mass splitting $\Delta M_{R}$ between two heavy RH neutrinos can be introduced (for example, by the radiative effect [104-106]), the resonant leptogenesis mechanism [107-109] can be successfully implemented

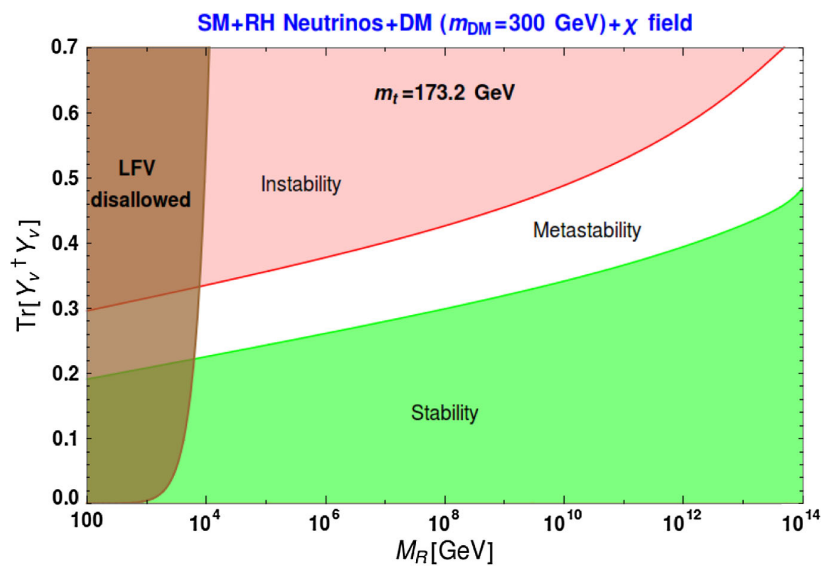

FIG. 21. LFV and absolute vacuum stability constraint on $\operatorname{Tr}\left[Y_{\nu}^{\dagger} Y_{\nu}\right]-M_{R}$ in the combined setup of $\mathrm{SM}+\mathrm{DM}+\mathrm{RH}$ eutrinos + $\chi$ field where $m_{\mathrm{DM}}=300 \mathrm{GeV}, m_{H_{2}}=300 \mathrm{GeV}, \sin \theta=0.2$, $\lambda_{\phi H}=0.06$, and $\lambda_{\chi \phi}=0.135$.
[110]. Apart from this, provided one can extend our vacuum stability analysis in the presence of nondegenerate RH neutrinos [43] with the DM and $\chi$ field, usual thermal leptogenesis can also be employed to explain the BAU of the Universe.

\section{CONCLUSIONS}

We have considered an extension of the SM by three RH neutrinos and two scalar singlets with an aim to study the EW vacuum stability in a framework that can incorporate a stable light DM within the reach of collider experiments and to explain the light neutrino mass. $A Z_{2} \times Z_{2}^{\prime}$ symmetry is imposed, in which $Z_{2}^{\prime}$ is broken from the vev of one of the scalars. It is known that with a real scalar singlet DM model present experimental limits by LUX 2016 and XENON 1T rule out DM mass below $m_{\mathrm{DM}}=500 \mathrm{GeV}$. Also, its presence does not modify the fate of the EW vacuum much and hence keeps it metastable only. Although metastability is acceptable, it, however, leaves some unwanted questions if we include primordial inflation in the picture. So, an absolute stability of the EW vacuum is more favorable. On the other hand, introduction of $\mathrm{RH}$ neutrinos would have large impact on the running of the Higgs quartic coupling due to the neutrino Yukawa interaction. Provided the neutrino Yukawa coupling is as large as $\mathcal{O}(1)$ or more, it can actually destabilize the EW vacuum. Hence, we have tried here to achieve the stability of the EW vacuum in presence of RH neutrinos and DM. We also planned to find the possibility of a light scalar DM below $500 \mathrm{GeV}$. For this purpose, we have introduced an additional scalar field that gets a vev. The other scalar among the two introduced does not get a vev and thereby is a good candidate for being a dark matter. The presence of the singlet with nonzero vev helps achieve the vacuum stability through a thresholdlike correction to $\lambda_{H}$. So, in this particular scenario, i.e., the SM extended by DM and three RH neutrinos plus one extra scalar, we have studied the Higgs vacuum stability issue, considering large Yukawa coupling and variation of $m_{t}$ within a $2 \sigma$ range of uncertainty. We have found the stability region in the $\operatorname{Tr}\left[Y_{\nu}^{\dagger} Y_{\nu}\right]-m_{t}$ plane has been significantly increased in the presence of $\chi$. Simultaneous mixing of this extra scalar with SM Higgs doublet ensures its involvement in the DM annihilations. This mixing is effectively controlled by the Higgs portal coupling of the scalar, which also enters into the running of the Higgs quartic coupling. Hence, an interplay between the two conditions, one to achieve the EW vacuum stability and the other to find a viable DM below $500 \mathrm{GeV}$, can actually constrain the parameters involved to some extent. Since the setup involves several new particles, finding their existence in future and ongoing experiments would be an interesting possibility for which to search. Here, we have considered the additional physical Higgs to be heavier than the SM one. One may also consider a scenario where the second physical Higgs is lighter than the SM Higgs discovered at $125 \mathrm{GeV}$. However, this case is not of very much interest in the 
present study, as following from Eq. (8), it can be seen that the effective Higgs quartic coupling becomes less than the $\mathrm{SM}$ one in this case, and this would not help make the EW vacuum stable. Also, the $\sin \theta$ allowed region for $m_{H_{2}}<$ $m_{H_{1}} / 2$ is almost excluded from the decay of $H_{2} \rightarrow H_{1} H_{1}$. Hence, we discard this possibility. One interesting extension of our work could be the study of a SM gauge extension in which the involvement of gauge bosons can modify our result. We keep it for a future study.

\section{ACKNOWLEDGMENTS}

P. G. and A.K.S. would like to thank MHRD, Government of India for research fellowship.

\section{APPENDIX: UNITARITY CONSTRAINTS}

In this section, we draw the perturbative unitarity limits on quartic couplings present in our model. The scattering amplitude for any $2 \rightarrow 2$ process can be expressed in terms of the Legendre polynomial as $[68,69]$

$$
\mathcal{M}^{2 \rightarrow 2}=16 \pi \sum_{l=0}^{\infty} a_{l}(2 l+1) P_{l}(\cos \theta),
$$

where $\theta$ is the scattering angle and $P_{l}(\cos \theta)$ is the Legendre polynomial of order $l$. In the high-energy limit, only the s-wave $(l=0)$ partial amplitude $a_{0}$ will determine the leading energy dependence of the scattering processes $[68,69]$. The unitarity constraint says

$$
\left|\operatorname{Re} \mathrm{a}_{0}\right|<1 / 2 \text {. }
$$

This constraint (A1) can be further translated to a bound on the scattering amplitude $\mathcal{M}[68,69]$ :

$$
|\mathcal{M}|<8 \pi
$$

In our proposed model, we have multiple possible $2 \rightarrow 2$ scattering processes. Therefore, we need to construct a matrix $\left(M_{i, j}^{2 \rightarrow 2}=\mathcal{M}_{i \rightarrow j}\right)$ considering all possible twoparticle states. Finally, we need to calculate the eigenvalues of $M$ and employ the bound as in Eq. (A2).

In the high-energy limit, we express the SM Higgs doublet as $H^{T}=\left(w^{+}, \frac{H^{0}+i z}{2}\right)$. Then, the scalar potential $(V)$ in Eq. (1) gives rise to 11 neutral combinations of twoparticle states,

$$
w^{+} w^{-}, \quad \frac{z z}{\sqrt{2}}, \quad \frac{H^{0} H^{0}}{\sqrt{2}}, \quad \frac{\chi \chi}{\sqrt{2}}, \quad \frac{\phi \phi}{\sqrt{2}}, \quad H^{0} \chi, \quad H^{0} \phi, \quad \chi \phi, \quad z H^{0}, \quad z \chi, \quad z \phi,
$$

and 4 singly charged two-particle states,

$$
w^{+} H^{0}, \quad w^{+} \chi, \quad w^{+} z, \quad w^{+} \phi .
$$

Hence, we can write the scattering amplitude matrix $(M)$ in block-diagonal form by decomposing it into a neutral and singly charged sector as

$$
M_{15 \times 15}=\left(\begin{array}{cc}
M_{11 \times 11}^{\mathrm{n}} & 0 \\
0 & M_{4 \times 4}^{s c}
\end{array}\right)
$$

The submatrices are provided below:

$$
M_{11 \times 11}^{n}=\left(\begin{array}{ccccccccccc}
4 \lambda_{H} & \sqrt{2} \lambda_{H} & \sqrt{2} \lambda_{H} & \frac{\lambda_{X H}}{\sqrt{2}} & \frac{\lambda_{\phi H}}{\sqrt{2}} & 0 & 0 & 0 & 0 & 0 & 0 \\
\sqrt{2} \lambda_{H} & 3 \lambda_{H} & \lambda_{H} & \frac{\lambda_{x H}}{2} & \frac{\lambda_{\phi H}}{2} & 0 & 0 & 0 & 0 & 0 & 0 \\
\sqrt{2} \lambda_{H} & \lambda_{H} & 3 \lambda_{H} & \frac{\lambda_{X H}}{2} & \frac{\lambda_{\phi H}}{2} & 0 & 0 & 0 & 0 & 0 & 0 \\
\frac{\lambda_{x H}}{\sqrt{2}} & \frac{\lambda_{X H}}{2} & \frac{\lambda_{\chi H}}{2} & \frac{\lambda_{x}}{2} & \frac{\lambda_{x \phi}}{2} & 0 & 0 & 0 & 0 & 0 & 0 \\
\frac{\lambda_{\phi H}}{\sqrt{2}} & \frac{\lambda_{\phi H}}{2} & \frac{\lambda_{\phi H}}{2} & \frac{\lambda_{x \phi}}{2} & \frac{\lambda_{\phi}}{2} & 0 & 0 & 0 & 0 & 0 & 0 \\
0 & 0 & 0 & 0 & 0 & \lambda_{\chi H} & 0 & 0 & 0 & 0 & 0 \\
0 & 0 & 0 & 0 & 0 & 0 & \lambda_{\phi H} & 0 & 0 & 0 & 0 \\
0 & 0 & 0 & 0 & 0 & 0 & 0 & \lambda_{\chi \phi} & 0 & 0 & 0 \\
0 & 0 & 0 & 0 & 0 & 0 & 0 & 0 & 2 \lambda_{H} & 0 & 0 \\
0 & 0 & 0 & 0 & 0 & 0 & 0 & 0 & 0 & \lambda_{\chi H} & 0 \\
0 & 0 & 0 & 0 & 0 & 0 & 0 & 0 & 0 & 0 & \lambda_{\phi H}
\end{array}\right),
$$




$$
M_{4 \times 4}^{s c}=\left(\begin{array}{cccc}
2 \lambda_{H} & 0 & 0 & 0 \\
0 & \lambda_{\chi H} & 0 & 0 \\
0 & 0 & 2 \lambda_{H} & 0 \\
0 & 0 & 0 & \lambda_{\phi H}
\end{array}\right) .
$$

The distinct eigenvalues of matrices (A6) and (A7) are

$$
2 \lambda_{H}, \quad \lambda_{\chi H}, \quad \lambda_{\phi H}, \quad \lambda_{\chi \phi} \text { and } x_{1,2,3},
$$

where $x_{1,2,3}$ are the roots of the following polynomial equation:

$$
\begin{aligned}
x^{3} & +x^{2}\left(-12 \lambda_{H}-\lambda \chi-\lambda \phi\right)+x\left(12 \lambda_{H} \lambda_{\chi}+12 \lambda_{H} \lambda_{\phi}-4 \lambda_{\chi H}^{2}-\lambda_{\chi_{\phi}}^{2}+\lambda_{\chi} \lambda_{\phi}-4 \lambda_{\phi H}^{2}\right) \\
& +12 \lambda_{H} \lambda_{\chi \phi}^{2}-12 \lambda_{H} \lambda_{\chi} \lambda_{\phi}+4 \lambda_{\chi H}^{2} \lambda_{\phi}+4 \lambda_{\chi} \lambda_{\phi H}^{2}-8 \lambda_{\chi H} \lambda_{\chi \phi} \lambda_{\phi H}=0 .
\end{aligned}
$$

Therefore, the unitarity constraints in the proposed setup are the following:

$$
\lambda_{H}<4 \pi, \quad \lambda_{\phi H}<8 \pi, \quad \lambda_{\chi H}<8 \pi, \quad \lambda_{\chi \phi}<8 \pi \quad \text { and } \quad x_{1,2,3}<16 \pi .
$$

[1] G. Aad et al. (ATLAS Collaboration), Phys. Lett. B 716, 1 (2012).

[2] S. Chatrchyan et al. (CMS Collaboration), Phys. Lett. B 716, 30 (2012).

[3] P. P. Giardino, K. Kannike, I. Masina, M. Raidal, and A. Strumia, J. High Energy Phys. 05 (2014) 046.

[4] K. A. Olive et al. (Particle Data Group Collaboration), Chin. Phys. C 38, 090001 (2014).

[5] G. Isidori, G. Ridolfi, and A. Strumia, Nucl. Phys. B609, 387 (2001).

[6] D. Buttazzo, G. Degrassi, P. P. Giardino, G. F. Giudice, F. Sala, A. Salvio, and A. Strumia, J. High Energy Phys. 12 (2013) 089.

[7] L. A. Anchordoqui, I. Antoniadis, H. Goldberg, X. Huang, D. Lust, T. R. Taylor, and B. Vlcek, J. High Energy Phys. 02 (2013) 074.

[8] Y. Tang, Mod. Phys. Lett. A 28, 1330002 (2013).

[9] G. Degrassi, S. Di Vita, J. Elias-Miro, J. R. Espinosa, G. F. Giudice, G. Isidori, and A. Strumia, J. High Energy Phys. 08 (2012) 098.

[10] J. Ellis, J. R. Espinosa, G. F. Giudice, A. Hoecker, and A. Riotto, Phys. Lett. B 679, 369 (2009).

[11] J. Elias-Miro, J. R. Espinosa, G. F. Giudice, G. Isidori, A. Riotto, and A. Strumia, Phys. Lett. B 709, 222 (2012).

[12] V. Branchina, E. Messina, and A. Platania, J. High Energy Phys. 09 (2014) 182.

[13] G. Degrassi, Nuovo Cimento C 037, 47 (2014).

[14] A. Kobakhidze and A. Spencer-Smith, Phys. Lett. B 722, 130 (2013).

[15] V. Silveira and A. Zee, Phys. Lett. 161B, 136 (1985).

[16] J. McDonald, Phys. Rev. D 50, 3637 (1994).
[17] J. M. Cline, K. Kainulainen, P. Scott, and C. Weniger, Phys. Rev. D 88, 055025 (2013); 92, 039906(E) (2015).

[18] W. L. Guo and Y. L. Wu, J. High Energy Phys. 10 (2010) 083.

[19] F. S. Queiroz and K. Sinha, Phys. Lett. B 735, 69 (2014).

[20] K. Ghorbani and H. Ghorbani, Phys. Rev. D 93, 055012 (2016).

[21] S. Bhattacharya, S. Jana, and S. Nandi, Phys. Rev. D 95, 055003 (2017).

[22] S. Bhattacharya, P. Poulose, and P. Ghosh, J. Cosmol. Astropart. Phys. 04 (2017) 043.

[23] J. A. Casas, D. G. Cerdeo, J. M. Moreno, and J. Quilis, J. High Energy Phys. 05 (2017) 036.

[24] D. S. Akerib et al. (LUX Collaboration), Phys. Rev. Lett. 118, 021303 (2017).

[25] E. Aprile et al. (XENON Collaboration), Phys. Rev. Lett. 119, 181301 (2017).

[26] P. Athron et al. (GAMBIT Collaboration), Eur. Phys. J. C 77, 568 (2017).

[27] E. Aprile et al. (XENON Collaboration), J. Cosmol. Astropart. Phys. 04 (2016) 027.

[28] J. Conrad, arXiv:1411.1925.

[29] N. Haba, K. Kaneta, and R. Takahashi, J. High Energy Phys. 04 (2014) 029.

[30] N. Khan and S. Rakshit, Phys. Rev. D 90, 113008 (2014).

[31] V. V. Khoze, C. McCabe, and G. Ro, J. High Energy Phys. 08 (2014) 026.

[32] M. Gonderinger, Y. Li, H. Patel, and M. J. Ramsey-Musolf, J. High Energy Phys. 01 (2010) 053.

[33] M. Gonderinger, H. Lim, and M. J. Ramsey-Musolf, Phys. Rev. D 86, 043511 (2012). 
[34] W. Chao, M. Gonderinger, and M. J. Ramsey-Musolf, Phys. Rev. D 86, 113017 (2012).

[35] E. Gabrielli, M. Heikinheimo, K. Kannike, A. Racioppi, M. Raidal, and C. Spethmann, Phys. Rev. D 89, 015017 (2014).

[36] C. S. Chen and Y. Tang, J. High Energy Phys. 04 (2012) 019.

[37] W. Rodejohann and H. Zhang, J. High Energy Phys. 06 (2012) 022.

[38] L. Delle Rose, C. Marzo, and A. Urbano, J. High Energy Phys. 12 (2015) 050.

[39] G. Bambhaniya, P. S. B. Dev, S. Goswami, S. Khan, and W. Rodejohann, Phys. Rev. D 95, 095016 (2017).

[40] M. Lindner, H. H. Patel, and B. Radovi, Phys. Rev. D 93, 073005 (2016).

[41] J. Chakrabortty, M. Das, and S. Mohanty, Mod. Phys. Lett. A 28, 1350032 (2013).

[42] A. Datta, A. Elsayed, S. Khalil, and A. Moursy, Phys. Rev. D 88, 053011 (2013).

[43] C. Coriano, L. Delle Rose, and C. Marzo, Phys. Lett. B 738, 13 (2014).

[44] J. N. Ng and A. de la Puente, Eur. Phys. J. C 76, 122 (2016).

[45] C. Bonilla, R. M. Fonseca, and J. W. F. Valle, Phys. Lett. B 756, 345 (2016).

[46] S. Khan, S. Goswami, and S. Roy, Phys. Rev. D 89, 073021 (2014).

[47] I. Garg, S. Goswami, K. N. Vishnudath, and N. Khan, Phys. Rev. D 96, 055020 (2017).

[48] E. Ma, Phys. Rev. D 73, 077301 (2006).

[49] H. Davoudiasl and I. M. Lewis, Phys. Rev. D 90, n033003 (2014).

[50] N. Chakrabarty, D. K. Ghosh, B. Mukhopadhyaya, and I. Saha, Phys. Rev. D 92, 015002 (2015).

[51] A. Abada and S. Nasri, Phys. Rev. D 88, 016006 (2013).

[52] A. Ahriche, A. Arhrib, and S. Nasri, J. High Energy Phys. 02 (2014) 042.

[53] N. Chakrabarty, U. K. Dey, and B. Mukhopadhyaya, J. High Energy Phys. 12 (2014) 166.

[54] D. Das and I. Saha, Phys. Rev. D 91, 095024 (2015).

[55] N. Chakrabarty and B. Mukhopadhyaya, Eur. Phys. J. C 77, 153 (2017).

[56] J. Elias-Miro, J. R. Espinosa, G. F. Giudice, H. M. Lee, and A. Strumia, J. High Energy Phys. 06 (2012) 031.

[57] N. Okada and Q. Shafi, Phys. Lett. B 747, 223 (2015).

[58] A. K. Saha and A. Sil, Phys. Lett. B 765, 244 (2017).

[59] K. Bhattacharya, J. Chakrabortty, S. Das, and T. Mondal, J. Cosmol. Astropart. Phys. 12 (2014) 001.

[60] Y. Ema, K. Mukaida, and K. Nakayama, Phys. Lett. B 761, 419 (2016).

[61] S. Baek, P. Ko, W. I. Park, and E. Senaha, J. High Energy Phys. 11 (2012) 116.

[62] G. Arcadi, M. Dutra, P. Ghosh, M. Lindner, Y. Mambrini, M. Pierre, S. Profumo, and F. S. Queiroz, Eur. Phys. J. C 78, 203 (2018).

[63] G. R. Dvali, Z. Tavartkiladze, and J. Nanobashvili, Phys. Lett. B 352, 214 (1995).

[64] A. Barroso, P. M. Ferreira, I. P. Ivanov, and R. Santos, J. High Energy Phys. 06 (2013) 045.

[65] A. Barroso, P. M. Ferreira, I. Ivanov, and R. Santos, arXiv:1305.1235.
[66] K. Kannike, Eur. Phys. J. C 72, 2093 (2012).

[67] J. Chakrabortty, P. Konar, and T. Mondal, Phys. Rev. D 89, 095008 (2014).

[68] J. Horejsi and M. Kladiva, Eur. Phys. J. C 46, 81 (2006).

[69] G. Bhattacharyya and D. Das, Pramana 87, 40 (2016).

[70] D. Lpez-Val and T. Robens, Phys. Rev. D 90, 114018 (2014).

[71] M. J. Strassler and K. M. Zurek, Phys. Lett. B 661, 263 (2008).

[72] V. Khachatryan et al. (CMS Collaboration), J. High Energy Phys. 10 (2015) 144.

[73] G. Aad et al. (ATLAS Collaboration), Eur. Phys. J. C 76, 45 (2016).

[74] S. Chatrchyan et al. (CMS Collaboration), Phys. Rev. D 89, 092007 (2014).

[75] CMS Collaboration, Report No. CMS-PAS-HIG-12-045, 2012.

[76] CMS Collaboration, Report No. CMS-PAS-HIG-13-003, 2013.

[77] T. Robens and T. Stefaniak, Eur. Phys. J. C 76, 268 (2016).

[78] ATLAS and CMS Collaborations, Report No. ATLASCONF-2015-044, 2015.

[79] P. A. R. Ade et al. (Planck Collaboration), Astron. Astrophys. 571, A16 (2014).

[80] G. Belanger, F. Boudjema, A. Pukhov, and A. Semenov, Comput. Phys. Commun. 185, 960 (2014).

[81] O. Lebedev, Eur. Phys. J. C 72, 2058 (2012).

[82] L. Basso, O. Fischer, and J. J. van Der Bij, Phys. Lett. B 730, 326 (2014).

[83] T. Robens and T. Stefaniak, Eur. Phys. J. C 75, 104 (2015).

[84] G. Chalons, D. Lopez-Val, T. Robens, and T. Stefaniak, Proc. Sci., ICHEP 2016 (2016) 1180.

[85] S. Dawson and I. M. Lewis, Phys. Rev. D 92, 094023 (2015).

[86] O. Fischer, Mod. Phys. Lett. A 32, 1750035 (2017).

[87] I. M. Lewis and M. Sullivan, Phys. Rev. D 96, 035037 (2017).

[88] J. M. Alarcon, J. Martin Camalich, and J. A. Oller, Phys. Rev. D 85, 051503 (2012).

[89] J. M. Alarcon, L. S. Geng, J. Martin Camalich, and J. A. Oller, Phys. Lett. B 730, 342 (2014).

[90] J. A. Casas and A. Ibarra, Nucl. Phys. B618, 171 (2001).

[91] W. 1. Guo, Z. z. Xing, and S. Zhou, Int. J. Mod. Phys. E 16, 1 (2007).

[92] Y. F. Pirogov and O. V. Zenin, Eur. Phys. J. C 10, 629 (1999).

[93] I. Esteban, M. C. Gonzalez-Garcia, M. Maltoni, I. MartinezSoler, and T. Schwetz, J. High Energy Phys. 01 (2017) 087.

[94] P. F. de Salas, D. V. Forero, C. A. Ternes, M. Tortola, and J. W. F. Valle, arXiv:1708.01186.

[95] A. Ilakovac and A. Pilaftsis, Nucl. Phys. B437, 491 (1995).

[96] D. Tommasini, G. Barenboim, J. Bernabeu, and C. Jarlskog, Nucl. Phys. B444, 451 (1995).

[97] D. N. Dinh, A. Ibarra, E. Molinaro, and S. T. Petcov, J. High Energy Phys. 08 (2012) 125; 09 (2013) 23.

[98] A. Ibarra, E. Molinaro, and S. T. Petcov, J. High Energy Phys. 09 (2010) 108.

[99] V. Tello, M. Nemevsek, F. Nesti, G. Senjanovic, and F. Vissani, Phys. Rev. Lett. 106, 151801 (2011). 
[100] M. Mitra, G. Senjanovic, and F. Vissani, Nucl. Phys. B856, 26 (2012).

[101] J. Chakrabortty, H. Z. Devi, S. Goswami, and S. Patra, J. High Energy Phys. 08 (2012) 008.

[102] P. S. Bhupal Dev, S. Goswami, and M. Mitra, Phys. Rev. D 91, 113004 (2015).

[103] P. S. Bhupal Dev, S. Goswami, M. Mitra, and W. Rodejohann, Phys. Rev. D 88, 091301 (2013).

[104] R. Gonzalez Felipe, F. R. Joaquim, and B. M. Nobre, Phys. Rev. D 70, 085009 (2004).
[105] K. Turzynski, Phys. Lett. B 589, 135 (2004).

[106] G. C. Branco, R. Gonzalez Felipe, F. R. Joaquim, and B. M. Nobre, Phys. Lett. B 633, 336 (2006).

[107] M. Flanz, E. A. Paschos, U. Sarkar, and J. Weiss, Phys. Lett. B 389, 693 (1996).

[108] A. Pilaftsis, Phys. Rev. D 56, 5431 (1997).

[109] A. Pilaftsis and T. E. J. Underwood, Nucl. Phys. B692, 303 (2004).

[110] G. C. Branco, R. Gonzalez Felipe, M. N. Rebelo, and H. Serodio, Phys. Rev. D 79, 093008 (2009). 